\title{
Multigene Phylogeny Reveals Haploanthostomella elaeidis gen. et sp. nov. and Familial Replacement of Endocalyx (Xylariales, Sordariomycetes, Ascomycota)
}

\author{
Sirinapa Konta 1,2,3 ${ }^{D}$, Kevin D. Hyde ${ }^{2}$, Prapassorn D. Eungwanichayapant ${ }^{3}$, Samantha C. Karunarathna 1,4,5, \\ Milan C. Samarakoon ${ }^{2}$, Jianchu Xu ${ }^{1,4,5}$, Lucas A. P. Dauner ${ }^{1}$, Sasith Tharanga Aluthwattha ${ }^{6,7}$, \\ Saisamorn Lumyong ${ }^{8,9}$ and Saowaluck Tibpromma ${ }^{1,4,5, *}$
}

check for updates

Citation: Konta, S.; Hyde, K.D.; Eungwanichayapant, P.D.;

Karunarathna, S.C.; Samarakoon, M.C.; Xu, J.; Dauner, L.A.P.; Aluthwattha, S.T.; Lumyong, S.; Tibpromma, S. Multigene Phylogeny Reveals Haploanthostomella elaeidis gen. et sp. nov. and Familial Replacement of Endocalyx (Xylariales, Sordariomycetes, Ascomycota). Life 2021, 11, 486. https://doi.org/ $10.3390 /$ life11060486

Academic Editors: Armin Mešić, Ivana Kušan and Laura Selbmann

Received: 30 March 2021

Accepted: 19 May 2021

Published: 26 May 2021

Publisher's Note: MDPI stays neutral with regard to jurisdictional claims in published maps and institutional affiliations.

Copyright: (C) 2021 by the authors. Licensee MDPI, Basel, Switzerland. This article is an open access article distributed under the terms and conditions of the Creative Commons Attribution (CC BY) license (https:/ / creativecommons.org/licenses/by/ $4.0 /)$
1 CAS Key Laboratory for Plant Diversity and Biogeography of East Asia, Kunming Institute of Botany, Chinese Academy of Sciences, Kunming 650201, China; sirinapakonta@gmail.com (S.K.); samanthakarunarathna@gmail.com (S.C.K.); jxu@mail.kib.ac.cn (J.X.); luke.dauner1@gmail.com (L.A.P.D.)

2 Center of Excellence in Fungal Research, Mae Fah Luang University, Chiang Rai 57100, Thailand; kdhyde3@gmail.com (K.D.H.); milan.chameera@yahoo.com (M.C.S.)

3 School of Science, Mae Fah Luang University, Chiang Rai 57100, Thailand; prapassorn@mfu.ac.th

4 World Agroforestry Centre, East and Central Asia, Kunming 650201, China

5 Centre for Mountain Futures, Kunming Institute of Botany, Kunming 650201, China

6 Guangxi Key Laboratory of Forest Ecology and Conservation, College of Forestry, Guangxi University, Daxuedonglu 100, Nanning 530004, China; aluthwattha@yahoo.com

7 State Key Laboratory of Conservation and Utilization of Subtropical Agro-Bioresources, College of Forestry, Guangxi University, Daxuedonglu 100, Nanning 530004, China

8 Research Center of Microbial Diversity and Sustainable Utilization, Faculty of Science, Chiang Mai University, Chiang Mai 50200, Thailand; saisamorn.1@cmu.ac.th

9 Academy of Science, The Royal Society of Thailand, Bangkok 10300, Thailand

* Correspondence: saowaluckfai@gmail.com (S.T.)

\begin{abstract}
During our investigation of palm fungi in Thailand, two interesting taxa from Elaeis guineensis and Metroxylon sagu (Arecaceae) were collected. Based on phylogenetic analyses of a combined dataset of ITS, LSU, $r p b 2$, and $t u b 2$ nucleotide sequences as well as unique morphological characteristics, we introduce the new genus Haploanthostomella within Xylariales, and a new species Endocalyx metroxyli. Additionally, in our study, the genus Endocalyx is transferred to the family Cainiaceae based on its brown conidia and molecular phylogenetic evidence.
\end{abstract}

Keywords: Apiosporaceae; Cainiaceae; fungi; palms; Thailand; Xylariales

\section{Introduction}

Palm trees represent a family of perennial lianas and consist of many diverse species worldwide, with the fossil record indicating around 65 million years of evolutionary history [1]. Microfungi on palms have been studied, but only a few have been analyzed using morphology and DNA sequence data. Several fungal species are currently unknown to science, with the total number estimated at somewhere between 2.2 and 3.8 million [2]. Thus, palms are a particularly interesting plant family for studying microfungi species unknown to science.

The subclass Xylariomycetidae has recently been updated to contain three orders (Amphisphaeriales, Delonicicolales, and Xylariales) and 35 families [3]. Recently, the family Induratiaceae was introduced in this subclass by Samarakoon et al. [4] with an updated phylogeny of Xylariales. Cainiaceae is a family of particular interest, as all members in this family tend to be found on monocotyledons, the majority of which are grasses [5]. In previous studies, Cainiaceae was accepted in the Xylariales [3,6]. Later, Hongsanan et al. [7], and Wijayawardene et al. [8] assigned Cainiaceae to the Xylariomycetidae as an incertae sedis family. 
The Xylariales is one of the largest orders and includes 15 families, 160 genera, and 52 genera incertae sedis [3]. Family Cainiaceae was introduced by Krug [9] to include species of Cainia with unique apical rings in the asci that consist of a series of rings and ascospores with longitudinal germ slits. An asexual morph of Cainiaceae was coelomycetous with black, scattered, immersed pycnidial conidiomata; hyaline, denticulate, sympodially proliferating conidiophores; hyaline, filiform, branched or simple, septate conidiogenous cells with one to three phialides; and hyaline, elongate fusiform, falcate to lunate, unicellular or septate conidia, with pointed ends [10]. At present, seven genera have been accepted into this family (Alishanica, Amphibambusa, Arecophila, Atrotorquata, Cainia, Longiappendispora, and Seynesia) $[3,11]$.

Since 2014, fungal research in Thailand has revealed a high diversity of novel species [12-14]. In this study, we found fungal species unknown to science from Thailand. The phylogeny results show that Endocalyx grouped within Cainiaceae, and so we transferred Endocalyx from Apiosporaceae (Amphisphaeriales) to Cainiaceae (Xylariales) based on both morphology and multigene phylogeny. We also introduce the new species Endocalyx metroxyli, collected from the economically important oil palm host (Elaeis guineensis). Lastly, we introduce the new genus Haploanthostomella associated with true sago palm (Metroxylon sagu).

\section{Materials and Methods}

\subsection{Collection, Isolation, and Identification}

Saprobic fungi growing on dead leaves, petioles and rachis of Elaeis guineensis and Metroxylon sagu were collected in Krabi and Surat Thani Provinces of Thailand, placed in ziplock bags and brought to the mycology laboratory at the Center of Excellence in Fungal Research, and morphological characteristics were observed. Specimens were examined following the methods provided by Konta et al. [15]. Single spore isolates were obtained following the method of Senanayake et al. [16], using malt extract agar (MEA) and incubating at $25-28{ }^{\circ} \mathrm{C}$ overnight. Germinating conidia were transferred to new MEA media and pure cultures were kept at $25-28{ }^{\circ} \mathrm{C}$. Specimens and cultures were deposited in the herbarium of Mae Fah Luang University (MFLU) and Mae Fah Luang University Culture Collection (MFLUCC), Chiang Rai, Thailand, respectively. Faces of Fungi and Index Fungorum numbers were registered as outlined in Jayasiri et al. [17] and Index Fungorum [18].

\subsection{DNA Extraction and Amplification (PCR)}

Genomic DNA was extracted from fruiting bodies of Haploanthostomella elaeidis and fungal mycelium of Endocalyx metroxyli. DNA extraction and amplification were followed Dissanayake et al. [19]. Konta et al.'s method [16] was followed for PCR amplification of ITS, LSU, SSU, tef1- $\alpha$ and $r p b 2$, while O'Donnell and Cigelnik's method [20] was followed for PCR amplification of the $t u b 2$ region. Amplification was done using the primers ITS5 and ITS4 for the internal transcribed spacer regions and intervening 5.8S rDNA (ITS), the primers LR5 and LR0R for the large subunit (LSU) rRNA gene, the primer pair fRPB2-5f and fRPB2-7cR for the RNA polymerase II second largest subunit (rpb2) gene, and the primers T1 and T22 for the partial gene $\beta$-tubulin ( $t u b 2)$. PCR amplifications were performed using $1 \times$ PCR buffer with $8.5 \mu \mathrm{L} \mathrm{ddH}_{2} \mathrm{O}, 12.5 \mu \mathrm{L} 2 \times$ Easy Taq PCR SuperMix (mixture of Easy Taq TM DNA Polymerase, dNTPs and optimized buffer (Beijing Trans Gen Biotech Co., Beijing, China)), $2 \mu \mathrm{L}$ of DNA template, and $1 \mu \mathrm{L}$ each of forward and reverse primers $(10 \mathrm{pM})$ in a final volume of $25 \mu \mathrm{L}$. The cycle conditions in the initiation step were started at $95{ }^{\circ} \mathrm{C}$ for $3 \mathrm{~min}$, followed by 35 cycles at $95{ }^{\circ} \mathrm{C}$ for $30 \mathrm{~s}, 55^{\circ} \mathrm{C}$ for $50 \mathrm{~s}, 72{ }^{\circ} \mathrm{C}$ for $30 \mathrm{~s}$ (for ITS, LSU); $95^{\circ} \mathrm{C}$ for $5 \mathrm{~min}$, followed by 35 cycles at $95^{\circ} \mathrm{C}$ for $1 \mathrm{~min}, 54{ }^{\circ} \mathrm{C}$ for $2 \mathrm{~min}, 72{ }^{\circ} \mathrm{C}$ for $1: 5 \mathrm{~min}$ (for $r p b 2$ ); $95^{\circ} \mathrm{C}$ for $5 \mathrm{~min}$, followed by 35 cycles at $94{ }^{\circ} \mathrm{C}$ for $1 \mathrm{~min}, 52{ }^{\circ} \mathrm{C}$ for $1 \mathrm{~min}, 72{ }^{\circ} \mathrm{C}$ for $1: 5 \mathrm{~min}$ (for $t u b 2$ ); a final elongation step at $72{ }^{\circ} \mathrm{C}$ for $10 \mathrm{~min}$ and a final hold at $4{ }^{\circ} \mathrm{C}$ were done as the last steps. Purification and sequencing were performed by 
Sangon Biotech Co., Shanghai, China. Consensus sequences were computed using SeqMan software, and new sequences generated in this study were deposited in GenBank (Table 1).

Table 1. Names, strain numbers and corresponding GenBank accession numbers of the taxa used in phylogenetic analyses, the ex-type strains are in bold.

\begin{tabular}{|c|c|c|c|c|c|c|c|c|}
\hline \multirow{2}{*}{ Order } & \multirow{2}{*}{ Family } & \multirow{2}{*}{ Species } & \multirow{2}{*}{ Strain No. } & \multicolumn{4}{|c|}{ GenBank Accession No. } & \multirow{2}{*}{ References } \\
\hline & & & & ITS & LSU & $r p b 2$ & $t u b 2$ & \\
\hline Amphisphaeriales & Apiosporaceae & $\begin{array}{l}\text { Arthrinium } \\
\text { balearicum }\end{array}$ & AP24118 & MK014869 & MK014836 & - & MK017946 & [21] \\
\hline Amphisphaeriales & Apiosporaceae & $\begin{array}{l}\text { Arthrinium } \\
\text { caricicola }\end{array}$ & CBS 145127 & MK014871 & MK014838 & - & MK017948 & [21] \\
\hline Amphisphaeriales & Apiosporaceae & $\begin{array}{c}\text { Arthrinium } \\
\text { hydei }\end{array}$ & CBS 114990 & KF144890 & KF144936 & - & KF144982 & [22] \\
\hline Amphisphaeriales & Apiosporaceae & $\begin{array}{l}\text { Arthrinium } \\
\text { phragmitis }\end{array}$ & CBS 135458 & KF144909 & KF144956 & - & KF145001 & {$[22]$} \\
\hline Amphisphaeriales & Apiosporaceae & $\begin{array}{c}\text { Arthrinium pseu- } \\
\text { dospegazzinii }\end{array}$ & CBS 102052 & KF144911 & KF144958 & - & KF145002 & [22] \\
\hline Amphisphaeriales & Apiosporaceae & $\begin{array}{l}\text { Nigrospora } \\
\text { aurantiaca }\end{array}$ & $\begin{array}{c}\text { CGMCC } \\
3.18130 \\
\end{array}$ & NR_153477 & NG_069394 & - & KY019465 & [23] \\
\hline Amphisphaeriales & Apiosporaceae & $\begin{array}{l}\text { Nigrospora } \\
\text { brasiliensis }\end{array}$ & CMM 1214 & KY569629 & - & - & MK720816 & [24] \\
\hline Amphisphaeriales & Apiosporaceae & $\begin{array}{c}\text { Nigrospora } \\
\text { zimmermanii }\end{array}$ & CBS 290.62 & KY385309 & - & KY806276 & KY385317 & [23] \\
\hline Amphisphaeriales & Beltraniaceae & $\begin{array}{l}\text { Beltrania } \\
\text { rhombica }\end{array}$ & $\begin{array}{l}\text { CBS } 123.58= \\
\text { IMI } 072432\end{array}$ & MH553990 & MH554209 & MH554899 & MH704631 & [25] \\
\hline Amphisphaeriales & Beltraniaceae & $\begin{array}{c}\text { Beltraniella } \\
\text { endiandrae }\end{array}$ & CBS 137976 & KJ869128 & KJ869185 & - & - & [26] \\
\hline Amphisphaeriales & Beltraniaceae & $\begin{array}{c}\text { Beltraniopsis } \\
\text { neolitseae }\end{array}$ & CBS 137974 & KJ869126 & KJ869183 & - & - & [26] \\
\hline Amphisphaeriales & Beltraniaceae & $\begin{array}{c}\text { Arecophila } \\
\text { bambusae }\end{array}$ & HKUCC 4794 & - & AF452038 & - & - & [27] \\
\hline Xylariales & Cainiaceae & $\begin{array}{l}\text { Alishanica } \\
\text { miscanthii }\end{array}$ & FU31025 & MK503821 & MK503827 & - & - & [3] \\
\hline Xylariales & Cainiaceae & $\begin{array}{c}\text { Amphibambusa } \\
\text { bambusicola }\end{array}$ & $\begin{array}{l}\text { MFLUCC } \\
11-0617\end{array}$ & KP744433 & KP744474 & - & - & [28] \\
\hline Xylariales & Cainiaceae & $\begin{array}{l}\text { Atrotorquata } \\
\text { lineata }\end{array}$ & HKUCC 3263 & AF009807 & - & - & - & Unpublished \\
\hline Xylariales & Cainiaceae & $\begin{array}{c}\text { Cainia } \\
\text { anthoxanthis }\end{array}$ & $\begin{array}{l}\text { MFLUCC } \\
15-0539\end{array}$ & KR092787 & KR092777 & - & - & [5] \\
\hline Xylariales & Cainiaceae & $\begin{array}{c}\text { Cainia } \\
\text { desmazieri }\end{array}$ & CAI & КТ949896 & КТ949896 & - & - & [29] \\
\hline Xylariales & Cainiaceae & Cainia globosa & $\begin{array}{c}\text { MFLUCC } \\
13-0663\end{array}$ & KX822127 & KX822123 & - & - & [30] \\
\hline Xylariales & Cainiaceae & Cainia graminis & CBS 136.62 & KR092793 & AF431949 & - & - & {$[5,31]$} \\
\hline Xylariales & Cainiaceae & $\begin{array}{l}\text { Longiappendispora } \\
\text { chromolaenae }\end{array}$ & $\begin{array}{l}\text { MFLUCC } \\
17-1485\end{array}$ & MT214370 & MT214464 & - & - & [11] \\
\hline Xylariales & Cainiaceae & $\begin{array}{l}\text { Endocalyx } \\
\text { cinctus }\end{array}$ & JCM 7946 & LC228648 & LC228704 & - & - & [32] \\
\hline Xylariales & Cainiaceae & $\begin{array}{l}\text { Endocalyx } \\
\text { metroxyli }\end{array}$ & $\begin{array}{l}\text { MFLUCC } \\
15-0723 \mathrm{~A}\end{array}$ & MT929162 & MT929313 & - & - & This study \\
\hline Xylariales & Cainiaceae & $\begin{array}{l}\text { Endocalyx } \\
\text { metroxyli }\end{array}$ & $\begin{array}{l}\text { MFLUCC } \\
15-0723 B\end{array}$ & МT929163 & MT929314 & - & MT928155 & This study \\
\hline Xylariales & Cainiaceae & $\begin{array}{l}\text { Endocalyx } \\
\text { metroxyli }\end{array}$ & $\begin{array}{l}\text { MFLUCC } \\
15-0723 C\end{array}$ & - & MT929315 & - & - & This study \\
\hline Xylariales & Cainiaceae & $\begin{array}{l}\text { Seynesia } \\
\text { erumpens }\end{array}$ & SMH 1291 & - & AF279410 & - & - & [33] \\
\hline Xylariales & Clypeosphaeriaceae & $\begin{array}{c}\text { Clypeosphaeria } \\
\text { mamillana }\end{array}$ & CBS 140735 & КT949897 & КТ949897 & MF489001 & MH704637 & {$[29,34]$} \\
\hline Xylariales & Coniocessiaceae & $\begin{array}{c}\text { Coniocessia } \\
\text { anandra }\end{array}$ & Co108 & GU553338 & GU553349 & - & - & [35] \\
\hline Xylariales & Coniocessiaceae & $\begin{array}{l}\text { Coniocessia } \\
\text { cruciformis }\end{array}$ & Co116 & GU553336 & GU553347 & - & - & {$[35]$} \\
\hline
\end{tabular}


Table 1. Cont.

\begin{tabular}{|c|c|c|c|c|c|c|c|c|}
\hline \multirow{2}{*}{ Order } & \multirow{2}{*}{ Family } & \multirow{2}{*}{ Species } & \multirow{2}{*}{ Strain No. } & \multicolumn{4}{|c|}{ GenBank Accession No. } & \multirow{2}{*}{ References } \\
\hline & & & & ITS & LSU & $r p b 2$ & $t u b 2$ & \\
\hline Xylariales & Coniocessiaceae & $\begin{array}{l}\text { Coniocessia } \\
\text { maxima }\end{array}$ & Co117 & GU553332 & GU553344 & - & - & [35] \\
\hline Xylariales & Coniocessiaceae & $\begin{array}{l}\text { Coniocessia } \\
\text { minima }\end{array}$ & Co111 & GU553334 & GU553345 & - & - & [35] \\
\hline Xylariales & Coniocessiaceae & $\begin{array}{c}\text { Coniocessia } \\
\text { nodulisporioides }\end{array}$ & CBS 281.77T & - & AJ875224 & - & - & [36] \\
\hline Xylariales & Coniocessiaceae & $\begin{array}{l}\text { Paraxylaria } \\
\text { rosacearum }\end{array}$ & TASM 6132 & MG828941 & MG829050 & - & - & [37] \\
\hline Xylariales & Diatrypaceae & $\begin{array}{c}\text { Allocryptovalsa } \\
\text { polyspora }\end{array}$ & $\begin{array}{c}\text { MFLUCC } \\
17-0364\end{array}$ & MF959500 & MF959503 & - & MG334556 & {$[38]$} \\
\hline Xylariales & Diatrypaceae & $\begin{array}{c}\text { Allodiatrype } \\
\text { arengae }\end{array}$ & $\begin{array}{c}\text { MFLUCC } \\
15-0713\end{array}$ & MN308411 & MN308402 & MN542886 & MN340297 & [39] \\
\hline Xylariales & Diatrypaceae & $\begin{array}{l}\text { Cryptovalsa } \\
\text { rabenhorstii }\end{array}$ & $\begin{array}{c}\text { CreI }=\text { CBS } \\
125574\end{array}$ & КС774567 & КС774567 & - & - & [40] \\
\hline Xylariales & Diatrypaceae & $\begin{array}{l}\text { Diatrype } \\
\text { disciformis }\end{array}$ & CBS 197.49 & - & DQ470964 & DQ470915 & - & [41] \\
\hline Xylariales & Diatrypaceae & $\begin{array}{l}\text { Diatrypella } \\
\text { verruciformis }\end{array}$ & UCROK1467 & JX144793 & - & - & JX174093 & [42] \\
\hline Xylariales & Diatrypaceae & Eutypa lata & CBS 208.87 & DQ006927 & MH873755 & - & DQ006969 & {$[43,44]$} \\
\hline Xylariales & Diatrypaceae & $\begin{array}{l}\text { Eutypella } \\
\text { caricae }\end{array}$ & EL5C & AJ302460 & - & - & - & [45] \\
\hline Xylariales & Diatrypaceae & $\begin{array}{l}\text { Halodiatrype } \\
\text { salinicola }\end{array}$ & $\begin{array}{l}\text { MFLUCC } \\
15-1277\end{array}$ & KX573915 & - & - & KX573932 & [46] \\
\hline Xylariales & Diatrypaceae & $\begin{array}{c}\text { Monosporascus } \\
\text { cannonballus }\end{array}$ & CMM3646 & JX971617 & - & - & - & Unpublished \\
\hline Xylariales & Diatrypaceae & $\begin{array}{l}\text { Neoeutypella } \\
\text { baoshanensis }\end{array}$ & $\begin{array}{l}\text { EL51C, CBS } \\
274.87\end{array}$ & AJ302460 & - & - & - & [45] \\
\hline Xylariales & Diatrypaceae & $\begin{array}{l}\text { Pedumispora } \\
\text { rhizophorae }\end{array}$ & BCC 44877 & KJ888853 & KJ888850 & - & - & [47] \\
\hline Xylariales & Diatrypaceae & $\begin{array}{l}\text { Peroneutypa } \\
\text { longiasca }\end{array}$ & $\begin{array}{l}\text { MFLUCC } \\
17-0371\end{array}$ & MF959502 & MF959505 & - & MG334558 & [38] \\
\hline Xylariales & Fasciatisporaceae & $\begin{array}{c}\text { Fasciatispora } \\
\text { arengae }\end{array}$ & $\begin{array}{l}\text { MFLUCC } \\
15-0326 a\end{array}$ & MK120275 & MK120300 & MK890794 & MK890793 & [48] \\
\hline Xylariales & Fasciatisporaceae & $\begin{array}{l}\text { Fasciatispora } \\
\text { calami }\end{array}$ & $\begin{array}{l}\text { MFLUCC } \\
15-0294\end{array}$ & - & MF459055 & - & MF459056 & [49] \\
\hline Xylariales & Fasciatisporaceae & $\begin{array}{c}\text { Fasciatispora } \\
\text { cocoes }\end{array}$ & $\begin{array}{l}\text { MFLUCC } \\
18-1445\end{array}$ & MN482680 & MN482675 & MN481517 & MN505154 & [13] \\
\hline Xylariales & Fasciatisporaceae & $\begin{array}{c}\text { Fasciatispora } \\
\text { nypae }\end{array}$ & $\begin{array}{l}\text { MFLUCC } \\
11-0382\end{array}$ & - & KP744484 & - & - & [28] \\
\hline Xylariales & Fasciatisporaceae & $\begin{array}{c}\text { Fasciatispora } \\
\text { petrakii }\end{array}$ & & - & AY083828 & - & - & Unpublished \\
\hline Xylariales & Graphostromataceae & $\begin{array}{c}\text { Biscogniauxia } \\
\text { nummularia }\end{array}$ & MUCL 51395 & KY610382 & KY610427 & KY624236 & KX271241 & [50] \\
\hline Xylariales & Graphostromataceae & Camillea obularia & ATCC 28093 & KY610384 & KY610429 & KY624238 & KX271243 & {$[50]$} \\
\hline Xylariales & Graphostromataceae & $\begin{array}{l}\text { Graphostroma } \\
\text { platystomum }\end{array}$ & CBS 270.87 & JX658535 & DQ836906 & KY624296 & HG934108 & [50-53] \\
\hline Xylariales & Graphostromataceae & $\begin{array}{l}\text { Obolarina } \\
\text { dryophila }\end{array}$ & MUCL 49882 & GQ428316 & GQ428316 & KY624284 & GQ428322 & {$[50,54]$} \\
\hline Xylariales & Hansfordiaceae & $\begin{array}{c}\text { Hansfordia } \\
\text { pulvinate }\end{array}$ & CBS 194.56 & MK442585 & MH869122 & KU684307 & - & [24] \\
\hline Xylariales & Hansfordiaceae & $\begin{array}{l}\text { Hansfordia } \\
\text { pulvinate }\end{array}$ & CBS 144422 & MK442587 & MK442527 & - & - & [24] \\
\hline Xylariales & Hypoxylaceae & $\begin{array}{l}\text { Annulohypoxylon } \\
\text { truncatum }\end{array}$ & CBS 140778 & KY610419 & KY610419 & KY624277 & KX376352 & {$[50,55]$} \\
\hline Xylariales & Hypoxylaceae & $\begin{array}{c}\text { Anthocanalis } \\
\text { sparti }\end{array}$ & $\begin{array}{c}\text { MFLUCC } \\
14-0010\end{array}$ & KP297394 & KP340536 & KP340522 & KP406605 & [54] \\
\hline Xylariales & Hypoxylaceae & $\begin{array}{l}\text { Anthostoma } \\
\text { decipiens }\end{array}$ & $\begin{array}{c}C D=C B S \\
133221\end{array}$ & KC774565 & КC774565 & - & - & {$[40]$} \\
\hline
\end{tabular}


Table 1. Cont.

\begin{tabular}{|c|c|c|c|c|c|c|c|c|}
\hline \multirow{2}{*}{ Order } & \multirow{2}{*}{ Family } & \multirow{2}{*}{ Species } & \multirow{2}{*}{ Strain No. } & \multicolumn{4}{|c|}{ GenBank Accession No. } & \multirow{2}{*}{ References } \\
\hline & & & & ITS & LSU & $r p b 2$ & $t u b 2$ & \\
\hline Xylariales & Hypoxylaceae & $\begin{array}{l}\text { Daldinia } \\
\text { concentrica }\end{array}$ & CBS 113277 & AY616683 & KY610434 & KY624243 & КС977274 & {$[50,56,57]$} \\
\hline Xylariales & Hypoxylaceae & $\begin{array}{l}\text { Durotheca } \\
\text { depressa }\end{array}$ & ВCC28073 & - & - & - & GQ160492 & [58] \\
\hline Xylariales & Hypoxylaceae & $\begin{array}{l}\text { Entonaema } \\
\text { liquescens }\end{array}$ & ATCC 46302 & KY610389 & KY610443 & KY624253 & KX271248 & [50] \\
\hline Xylariales & Hypoxylaceae & $\begin{array}{l}\text { Hypomontagnella } \\
\text { monticulosa }\end{array}$ & MUCL 54604 & KY610404 & KY610487 & KY624305 & KX271273 & {$[50]$} \\
\hline Xylariales & Hypoxylaceae & $\begin{array}{l}\text { Hypoxylon } \\
\text { fragiforme }\end{array}$ & MUCL 51264 & КС477229 & KM186295 & KM186296 & KX271282 & {$[50,59,60]$} \\
\hline Xylariales & Hypoxylaceae & $\begin{array}{l}\text { Jackrogersella } \\
\text { multiformis }\end{array}$ & CBS 119016 & КС477234 & KY610473 & KY624290 & KX271262 & {$[50,55,57]$} \\
\hline Xylariales & Hypoxylaceae & $\begin{array}{c}\text { Pyrenomyxa } \\
\text { morganii }\end{array}$ & CBS 116990T & AM749920 & - & - & - & [61] \\
\hline Xylariales & Hypoxylaceae & Pyrenomyxa picea & ILLS 58257 & - & EF562506 & - & - & {$[62]$} \\
\hline Xylariales & Hypoxylaceae & $\begin{array}{l}\text { Pyrenopolyporus } \\
\text { hunteri }\end{array}$ & MUCL 52673 & KY610421 & KY610472 & KY624309 & KU159530 & {$[50,55]$} \\
\hline Xylariales & Hypoxylaceae & $\begin{array}{l}\text { Rhopalostroma } \\
\text { indicum }\end{array}$ & CBS 113035 & MH862909 & MH874483 & - & - & [44] \\
\hline Xylariales & Hypoxylaceae & $\begin{array}{l}\text { Thamnomyces } \\
\text { dendroidea }\end{array}$ & CBS 123578 & FN428831 & KY610467 & KY624232 & KY624313 & {$[50,63]$} \\
\hline Xylariales & Hypoxylaceae & $\begin{array}{c}\text { Thuemenella } \\
\text { cubispora }\end{array}$ & CBS 119807 & JX658531 & EF562508 & - & - & [62] \\
\hline Xylariales & Hypoxylaceae & Phylacia sagrana & CBS 119992 & AM749919 & - & - & - & {$[61]$} \\
\hline Xylariales & Hypoxylaceae & $\begin{array}{l}\text { Pyrenopolyporus } \\
\text { symphyon }\end{array}$ & TBRC: 8873 & MH938529 & MH938538 & MK165428 & MK165419 & [64] \\
\hline Xylariales & Induratiaceae & $\begin{array}{c}\text { Emarcea } \\
\text { castanopsidicola }\end{array}$ & CBS 117105 & MK762710 & MK762717 & MK791285 & MK776962 & [64] \\
\hline Xylariales & Induratiaceae & $\begin{array}{c}\text { Emarcea } \\
\text { eucalyptigena }\end{array}$ & CBS 139908 & MK762711 & MK762718 & MK791286 & MK776963 & {$[64]$} \\
\hline Xylariales & Induratiaceae & $\begin{array}{c}\text { Induratia } \\
\text { fengyangensis }\end{array}$ & CGMCC 2862 & HM034856 & HM034859 & HM034849 & HМ034843 & {$[65]$} \\
\hline Xylariales & Induratiaceae & $\begin{array}{l}\text { Induratia } \\
\text { thailandica }\end{array}$ & $\begin{array}{l}\text { MFLUCC } \\
17-2669\end{array}$ & MK762707 & MK762714 & MK791283 & MK776960 & {$[64]$} \\
\hline Xylariales & Lopadostomataceae & $\begin{array}{c}\text { Creosphaeria } \\
\text { sassafras }\end{array}$ & STMA 14087 & KY610411 & KY610468 & KY624265 & KX271258 & {$[50]$} \\
\hline Xylariales & Lopadostomataceae & $\begin{array}{l}\text { Lopadostoma } \\
\text { turgidum }\end{array}$ & CBS 133207 & KC774618 & KC774618 & КС774563 & MF489024 & {$[29,40]$} \\
\hline Xylariales & Microdochiaceae & Idriella lunata & MUCL 4103 & KC775734 & КС775709 & - & - & [66] \\
\hline Xylariales & Microdochiaceae & Idriella lunata & CBS 204.56 & KP859044 & KP858981 & - & - & [67] \\
\hline Xylariales & Microdochiaceae & $\begin{array}{l}\text { Microdochium } \\
\text { phragmitis }\end{array}$ & CBS 423.78 & KP859012 & KP858948 & KP859121 & KP859076 & [67] \\
\hline Xylariales & Polystigmataceae & $\begin{array}{l}\text { Polystigma } \\
\text { fulvum }\end{array}$ & MFLU 18-0261 & MK429738 & MK429727 & - & - & [68] \\
\hline Xylariales & Polystigmataceae & $\begin{array}{c}\text { Polystigma } \\
\text { rubrum }\end{array}$ & MFLU 15-3091 & KY594023 & MF981079 & - & - & [68] \\
\hline Xylariales & Requienellaceae & $\begin{array}{c}\text { Acrocordiella } \\
\text { occulta }\end{array}$ & RS9 & KT949893 & KT949893 & - & - & [29] \\
\hline Xylariales & Requienellaceae & $\begin{array}{c}\text { Acrocordiella } \\
\text { omanensis }\end{array}$ & SQUCC 15091 & MG584568 & MG584570 & - & - & [69] \\
\hline Xylariales & Requienellaceae & $\begin{array}{l}\text { Requienella } \\
\text { fraxini }\end{array}$ & RS2 & KT949909 & КT949909 & - & - & [29] \\
\hline Xylariales & Requienellaceae & $\begin{array}{l}\text { Requienella } \\
\text { seminuda }\end{array}$ & $\begin{array}{c}\mathrm{RS} 12=\mathrm{CBS} \\
140502\end{array}$ & KT949912 & KT949912 & MK523300 & - & {$[29,64]$} \\
\hline Xylariales & Xylariaceae & $\begin{array}{c}\text { Abieticola } \\
\text { koreana }\end{array}$ & EML-F0010-1 & JN977612 & JQ014618 & KP792128 & KP792126 & [70] \\
\hline Xylariales & Xylariaceae & $\begin{array}{l}\text { Amphirosellinia } \\
\text { nigrospora }\end{array}$ & $\begin{array}{l}\text { HAST } \\
91092308\end{array}$ & GU322457 & - & GQ848340 & GQ495951 & [71] \\
\hline Xylariales & Xylariaceae & $\begin{array}{l}\text { Anthostomella } \\
\text { formosa }\end{array}$ & $\begin{array}{l}\text { MFLUCC } \\
14-0170\end{array}$ & KР297403 & KР340544 & KP340531 & KP406614 & [59] \\
\hline
\end{tabular}


Table 1. Cont.

\begin{tabular}{|c|c|c|c|c|c|c|c|c|}
\hline \multirow{2}{*}{ Order } & \multirow{2}{*}{ Family } & \multirow{2}{*}{ Species } & \multirow{2}{*}{ Strain No. } & \multicolumn{4}{|c|}{ GenBank Accession No. } & \multirow{2}{*}{ References } \\
\hline & & & & ITS & LSU & $r p b 2$ & $t u b 2$ & \\
\hline Xylariales & Xylariaceae & $\begin{array}{l}\text { Anthostomella } \\
\text { helicofissa }\end{array}$ & $\begin{array}{l}\text { MFLUCC } \\
14-0173\end{array}$ & KP297406 & KР340547 & KР340534 & KP406617 & [59] \\
\hline Xylariales & Xylariaceae & $\begin{array}{c}\text { Anthostomella } \\
\text { obesa }\end{array}$ & $\begin{array}{c}\text { MFLUCC } \\
14-0171\end{array}$ & KP297405 & KР340546 & KP340533 & KP406616 & [59] \\
\hline Xylariales & Xylariaceae & $\begin{array}{l}\text { Anthostomella } \\
\text { pseudobambusi- } \\
\text { cola }\end{array}$ & $\begin{array}{l}\text { MFLUCC } \\
15-0192\end{array}$ & KU940153 & KU863141 & - & - & [72] \\
\hline Xylariales & Xylariaceae & $\begin{array}{c}\text { Anthostomelloides } \\
\text { brabeji }\end{array}$ & CBS 110128 & EU552098 & EU552098 & - & - & [73] \\
\hline Xylariales & Xylariaceae & $\begin{array}{l}\text { Anthostomelloides } \\
\text { forlicesenica }\end{array}$ & $\begin{array}{l}\text { MFLUCC } \\
14-0558\end{array}$ & KP297397 & KP340539 & - & KP406608 & [66] \\
\hline Xylariales & Xylariaceae & $\begin{array}{l}\text { Anthostomelloides } \\
\text { krabiensis }\end{array}$ & $\begin{array}{l}\text { MFLUCC } \\
15-0678\end{array}$ & KX305927 & KX305928 & KX305929 & - & [30] \\
\hline Xylariales & Xylariaceae & $\begin{array}{l}\text { Anthostomelloides } \\
\text { leucospermi }\end{array}$ & CBS:110126 & EU552100 & - & - & - & [73] \\
\hline Xylariales & Xylariaceae & $\begin{array}{l}\text { Anthostomelloides } \\
\text { proteae }\end{array}$ & CBS 110127 & EU552101 & - & - & - & [73] \\
\hline Xylariales & Xylariaceae & $\begin{array}{l}\text { Astrocystis } \\
\text { mirabilis }\end{array}$ & $\begin{array}{l}94070803 \\
\text { HAST }\end{array}$ & GU322448 & - & GQ844835 & GQ495941 & [71] \\
\hline Xylariales & Xylariaceae & $\begin{array}{c}\text { Brunneiperidium } \\
\text { gracilentum }\end{array}$ & $\begin{array}{c}\text { MFLUCC } \\
14-0011 \\
\text { Ex-type }\end{array}$ & KP297400 & KP340542 & KР340528 & KP406611 & [66] \\
\hline Xylariales & Xylariaceae & $\begin{array}{l}\text { Collodiscula } \\
\text { japonica }\end{array}$ & CBS 124266 & JF440974 & JF440974 & KY624273 & KY624316 & {$[50,74]$} \\
\hline Xylariales & Xylariaceae & $\begin{array}{c}\text { Coniolariella } \\
\text { gamsii }\end{array}$ & $\begin{array}{c}\text { Co27IRAN } \\
\text { 842C, } \\
\text { CBS114379 (T) }\end{array}$ & GU553325 & GU553329 & - & - & [35] \\
\hline Xylariales & Xylariaceae & $\begin{array}{l}\text { Entalbostroma } \\
\text { erumpens }\end{array}$ & ICMP 21152 & KX258206 & - & KX258204 & KX258205 & [75] \\
\hline Xylariales & Xylariaceae & $\begin{array}{l}\text { Entoleuca } \\
\text { mammata }\end{array}$ & J.D.R. 100 & GU300072 & - & GQ844782 & GQ470230 & [71] \\
\hline Xylariales & Xylariaceae & $\begin{array}{c}\text { Euepixylon } \\
\text { sphaeriostomum }\end{array}$ & J.D.R. 261 & GU292821 & - & GQ844774 & GQ470224 & [71] \\
\hline Xylariales & Xylariaceae & $\begin{array}{l}\text { Halorosellinia } \\
\text { oceanica }\end{array}$ & SGLAf82 & EU715635 & - & - & - & [76] \\
\hline Xylariales & Xylariaceae & $\begin{array}{l}\text { Hypocopra } \\
\text { rostrata }\end{array}$ & NRRL 66178 & KM067909 & - & - & - & [77] \\
\hline Xylariales & Xylariaceae & $\begin{array}{l}\text { Hypocreodendron } \\
\text { sanguineum }\end{array}$ & J.D.R. 169 & GU322433 & - & GQ844819 & GQ487710 & [71] \\
\hline Xylariales & Xylariaceae & $\begin{array}{l}\text { Kretzschmaria } \\
\text { clavus }\end{array}$ & YMJ 114 & EF026126 & - & GQ844789 & EF025611 & {$[71,78]$} \\
\hline Xylariales & Xylariaceae & $\begin{array}{l}\text { Linosporopsis } \\
\text { ischnotheca }\end{array}$ & $\begin{array}{c}\text { LIF1 = CBS } \\
145761\end{array}$ & MN818952 & MN818952 & MN820708 & MN820715 & [79] \\
\hline Xylariales & Xylariaceae & $\begin{array}{l}\text { Lunatiannulus } \\
\text { irregularis }\end{array}$ & $\begin{array}{l}\text { MFLUCC } \\
14-0014\end{array}$ & KP297398 & KP340540 & KР340526 & KP406609 & [57] \\
\hline Xylariales & Xylariaceae & Nemania serpens & CBS 679.86 & KU683765 & - & KU684284 & KU684188 & [80] \\
\hline Xylariales & Xylariaceae & $\begin{array}{c}\text { Neoxylaria } \\
\text { arengae }\end{array}$ & $\begin{array}{l}\text { MFLUCC } \\
15-0292\end{array}$ & MT496747 & - & MT502418 & - & [81] \\
\hline Xylariales & Xylariaceae & $\begin{array}{l}\text { Podosordaria } \\
\text { mexicana }\end{array}$ & WSP 176 & GU324762 & - & GQ853039 & GQ844840 & [71] \\
\hline Xylariales & Xylariaceae & $\begin{array}{l}\text { Poronia } \\
\text { punctata }\end{array}$ & CBS 656.78 & KT281904 & KY610496 & KY624278 & KX271281 & {$[5,50]$} \\
\hline Xylariales & Xylariaceae & Rosellinia aquila & MUCL 51703 & KY610392 & KY610460 & KY624285 & KX271253 & [50] \\
\hline Xylariales & Xylariaceae & $\begin{array}{l}\text { Rostrohypoxylon } \\
\text { terebratum }\end{array}$ & CBS 119137 & DQ631943 & DQ840069 & DQ631954 & DQ840097 & {$[82,83]$} \\
\hline Xylariales & Xylariaceae & $\begin{array}{c}\text { Ruwenzoria } \\
\text { pseudoannulata }\end{array}$ & MUCL 51394 & KY610406 & KY610494 & KY624286 & KX271278 & [50] \\
\hline Xylariales & Xylariaceae & $\begin{array}{l}\text { Sarcoxylon } \\
\text { compunctum }\end{array}$ & CBS 359.61 & KT281903 & KY610462 & KY624230 & KX271255 & {$[5,50]$} \\
\hline
\end{tabular}


Table 1. Cont.

\begin{tabular}{|c|c|c|c|c|c|c|c|c|}
\hline \multirow{2}{*}{ Order } & \multirow{2}{*}{ Family } & \multirow{2}{*}{ Species } & \multirow{2}{*}{ Strain No. } & \multicolumn{4}{|c|}{ GenBank Accession No. } & \multirow{2}{*}{ References } \\
\hline & & & & ITS & LSU & $r p b 2$ & tub2 & \\
\hline Xylariales & Xylariaceae & $\begin{array}{l}\text { Stilbohypoxylon } \\
\text { elaeicola }\end{array}$ & Y.M.J. 173 & EF026148 & - & GQ844826 & EF025616 & [71] \\
\hline Xylariales & Xylariaceae & $\begin{array}{c}\text { Stilbohypoxylon } \\
\text { elaeidis }\end{array}$ & $\begin{array}{l}\text { MFLUCC } \\
15-0295 a\end{array}$ & MT496745 & MT496755 & MT502416 & MT502420 & [81] \\
\hline Xylariales & Xylariaceae & $\begin{array}{l}\text { Stilbohypoxylon } \\
\text { quisquiliarum }\end{array}$ & Y.M.J. 172 & EF026119 & - & GQ853020 & EF025605 & [71] \\
\hline Xylariales & Xylariaceae & $\begin{array}{l}\text { Vamsapriya } \\
\text { bambusicola }\end{array}$ & $\begin{array}{l}\text { MFLUCC } \\
11-0477\end{array}$ & KM462835 & KM462836 & KM462834 & KM462833 & [84] \\
\hline Xylariales & Xylariaceae & $\begin{array}{l}\text { Vamsapriya bre- } \\
\text { viconidiophora }\end{array}$ & $\begin{array}{l}\text { MFLUCC } \\
14-0436 \\
\end{array}$ & MF621584 & MF621588 & - & - & [39] \\
\hline Xylariales & Xylariaceae & $\begin{array}{l}\text { Vamsapriya } \\
\text { indica }\end{array}$ & $\begin{array}{l}\text { MFLUCC } \\
12-0544\end{array}$ & KM462839 & KM462840 & KM462841 & KM462838 & [84] \\
\hline Xylariales & Xylariaceae & $\begin{array}{c}\text { Vamsapriya } \\
\text { khunkonensis }\end{array}$ & $\begin{array}{l}\text { MFLUCC } \\
11-0475\end{array}$ & KM462830 & KM462831 & KM462829 & KM462828 & {$[84]$} \\
\hline Xylariales & Xylariaceae & $\begin{array}{l}\text { Vamsapriya } \\
\text { yunnana }\end{array}$ & $\begin{array}{l}\text { KUMCC } \\
18-0008\end{array}$ & MG833874 & MG833873 & MG833875 & - & [85] \\
\hline Xylariales & Xylariaceae & $\begin{array}{l}\text { Virgaria } \\
\text { boninensis }\end{array}$ & JCM 18624 & AB740956 & AB740960 & - & - & [86] \\
\hline Xylariales & Xylariaceae & Virgaria nigra & CBS 128006 & MH864744 & MH876180 & - & - & {$[44]$} \\
\hline Xylariales & Xylariaceae & Xylaria hypoxylon & CBS 122620 & KY610407 & KY610495 & KY624231 & KX271279 & {$[50,87]$} \\
\hline $\begin{array}{c}\text { Sordariomycetes } \\
\text { genera } \\
\text { incertae sedis }\end{array}$ & $\begin{array}{l}\text { Xylariales genera } \\
\text { incertae sedis }\end{array}$ & $\begin{array}{c}\text { Melanographium } \\
\text { phoenicis }\end{array}$ & $\begin{array}{l}\text { MFLUCC } \\
18-1481\end{array}$ & MN482677 & MN482678 & - & - & [13] \\
\hline $\begin{array}{c}\text { Sordariomycetes } \\
\text { genera } \\
\text { incertae sedis }\end{array}$ & $\begin{array}{l}\text { Xylariales genera } \\
\text { incertae sedis }\end{array}$ & $\begin{array}{l}\text { Ceratocladium } \\
\text { microspermum }\end{array}$ & CBS126092 & MH864077 & MH875534 & - & - & {$[44]$} \\
\hline Xylariales & $\begin{array}{l}\text { Xylariales genera } \\
\text { incertae sedis }\end{array}$ & $\begin{array}{l}\text { Ascotricha } \\
\text { chartarum }\end{array}$ & CBS 234.97 & KF893284 & - & - & KF893271 & [88] \\
\hline Xylariales & $\begin{array}{l}\text { Xylariales genera } \\
\text { incertae sedis }\end{array}$ & $\begin{array}{l}\text { Ascotricha } \\
\text { longipila }\end{array}$ & $\begin{array}{l}\text { OUCMBI110118 } \\
\text { (T) }\end{array}$ & KC503896 & - & - & KF893265 & [88] \\
\hline Xylariales & $\begin{array}{l}\text { Xylariales genera } \\
\text { incertae sedis }\end{array}$ & $\begin{array}{l}\text { Ascotricha } \\
\text { lusitanica }\end{array}$ & $\begin{array}{l}\text { CBS } 462.70 \\
\text { (IT) }\end{array}$ & KF893289 & - & - & KF893275 & [88] \\
\hline Xylariales & $\begin{array}{l}\text { Xylariales genera } \\
\text { incertae sedis }\end{array}$ & $\begin{array}{l}\text { Ascotricha } \\
\text { parvispora }\end{array}$ & $\begin{array}{l}\text { OUCMBI110001 } \\
\text { (T) }\end{array}$ & JX014298 & - & - & KF893267 & [88] \\
\hline Xylariales & $\begin{array}{l}\text { Xylariales genera } \\
\text { incertae sedis }\end{array}$ & $\begin{array}{l}\text { Ascotricha } \\
\text { sinuosa }\end{array}$ & $\begin{array}{l}\text { OUCMBI101190 } \\
\text { (T) }\end{array}$ & JX014299 & - & - & KF893266 & [88] \\
\hline Xylariales & $\begin{array}{l}\text { Xylariales genera } \\
\text { incertae sedis }\end{array}$ & $\begin{array}{l}\text { Alloanthostomella } \\
\text { rubicola }\end{array}$ & $\begin{array}{l}\text { MFLUCC } \\
14-0175 \\
\end{array}$ & KP297407 & KР340548 & KР340535 & КР406618 & [89] \\
\hline Xylariales & $\begin{array}{l}\text { Xylariales genera } \\
\text { incertae sedis }\end{array}$ & $\begin{array}{l}\text { Circinotrichum } \\
\text { cycadis }\end{array}$ & СРC 17285 & KJ869121 & KJ869178 & - & - & [26] \\
\hline Xylariales & $\begin{array}{l}\text { Xylariales genera } \\
\text { incertae sedis }\end{array}$ & $\begin{array}{l}\text { Circinotrichum } \\
\text { maculiforme }\end{array}$ & СРC 24566 & KR611874 & KR611895 & - & - & [90] \\
\hline Xylariales & $\begin{array}{l}\text { Xylariales genera } \\
\text { incertae sedis }\end{array}$ & $\begin{array}{l}\text { Circinotrichum } \\
\text { papakurae }\end{array}$ & CBS 101373 & KR611876 & KR611897 & - & - & [90] \\
\hline Xylariales & $\begin{array}{l}\text { Xylariales genera } \\
\text { incertae sedis }\end{array}$ & $\begin{array}{l}\text { Circinotrichum } \\
\text { sinense }\end{array}$ & & KY994106 & KY994107 & - & - & [91] \\
\hline Xylariales & $\begin{array}{l}\text { Xylariales genera } \\
\text { incertae sedis }\end{array}$ & $\begin{array}{l}\text { Gyrothrix } \\
\text { eucalypti }\end{array}$ & СРС 36066 & MN562109 & MN567617 & - & - & [92] \\
\hline Xylariales & $\begin{array}{l}\text { Xylariales genera } \\
\text { incertae sedis }\end{array}$ & Gyrothrix inops & BE108 & KC775746 & КC775721 & - & - & [66] \\
\hline Xylariales & $\begin{array}{l}\text { Xylariales genera } \\
\text { incertae sedis }\end{array}$ & Gyrothrix oleae & СРС 37069 & MN562136 & MN567643 & - & - & [92] \\
\hline Xylariales & $\begin{array}{l}\text { Xylariales genera } \\
\text { incertae sedis }\end{array}$ & $\begin{array}{l}\text { Gyrothrix } \\
\text { ramosa }\end{array}$ & MUCL54061 & КC775747 & КC775722 & - & - & [66] \\
\hline Xylariales & $\begin{array}{l}\text { Xylariales genera } \\
\text { incertae sedis }\end{array}$ & $\begin{array}{l}\text { Haploanthostomell } \\
\text { elaeidis }\end{array}$ & ${ }^{a}$ MFLU 20-0522 & MT929161 & MT929312 & MT928154 & - & This study \\
\hline
\end{tabular}


Table 1. Cont.

\begin{tabular}{|c|c|c|c|c|c|c|c|c|}
\hline \multirow{2}{*}{ Order } & \multirow{2}{*}{ Family } & \multirow{2}{*}{ Species } & \multirow{2}{*}{ Strain No. } & \multicolumn{4}{|c|}{ GenBank Accession No. } & \multirow{2}{*}{ References } \\
\hline & & & & ITS & LSU & $r p b 2$ & $t u b 2$ & \\
\hline Xylariales & $\begin{array}{l}\text { Xylariales genera } \\
\text { incertae sedis }\end{array}$ & $\begin{array}{l}\text { Neoanthostomella } \\
\text { pseudostromatica }\end{array}$ & $\begin{array}{l}\text { MFLUCC } \\
11-0610\end{array}$ & KU940158 & KU863146 & - & - & [72] \\
\hline Xylariales & $\begin{array}{l}\text { Xylariales genera } \\
\text { incertae sedis }\end{array}$ & $\begin{array}{c}\text { Neoanthostomella } \\
\text { viticola }\end{array}$ & $\begin{array}{l}\text { MFLUCC } \\
16-0243\end{array}$ & KX505957 & KX505958 & KX789496 & KX789495 & [89] \\
\hline Xylariales & $\begin{array}{l}\text { Xylariales genera } \\
\text { incertae sedis }\end{array}$ & $\begin{array}{l}\text { Pseudoanthostomella } \\
\text { conorum }\end{array}$ & CBS 119333 & EU552099 & - & - & - & [73] \\
\hline Xylariales & $\begin{array}{l}\text { Xylariales genera } \\
\text { incertae sedis }\end{array}$ & $\begin{array}{l}\text { Pseudoanthostomella } \\
\text { delitescens }\end{array}$ & $\begin{array}{c}\text { MFLUCC } \\
16-0477\end{array}$ & KX533451 & KX533452 & KX789491 & KX789490 & [89] \\
\hline Xylariales & $\begin{array}{l}\text { Xylariales genera } \\
\text { incertae sedis }\end{array}$ & $\begin{array}{l}\text { Pseudoanthostomella } \\
\text { pini-nigrae }\end{array}$ & $\begin{array}{c}\text { MFLUCC } \\
16-0478\end{array}$ & KX533453 & KX533454 & KX789492 & - & [89] \\
\hline Xylariales & $\begin{array}{l}\text { Xylariales genera } \\
\text { incertae sedis }\end{array}$ & $\begin{array}{l}\text { Pseudoanthostomella } \\
\text { sepelibilis }\end{array}$ & & AY908989 & AY875645 & - & - & Unpublished \\
\hline Xylariales & $\begin{array}{l}\text { Xylariales genera } \\
\text { incertae sedis }\end{array}$ & $\begin{array}{l}\text { Xenoanthostomella } \\
\text { chromolaenae }\end{array}$ & $\begin{array}{c}\text { MFLUCC } \\
17-1484 \\
\end{array}$ & MN638863 & MN638848 & - & - & [3] \\
\hline Xylariales & Zygosporiaceae & $\begin{array}{l}\text { Zygosporium } \\
\text { oscheoides }\end{array}$ & $\begin{array}{l}\text { MFLUCC } \\
14-0402 \\
\end{array}$ & MF621585 & MF621589 & - & - & [93] \\
\hline Xylariales & Zygosporiaceae & $\begin{array}{l}\text { Zygosporium } \\
\text { minus }\end{array}$ & HKAS99625 & MF621586 & MF621590 & - & - & [93] \\
\hline
\end{tabular}

\subsection{Phylogenetic Analyses}

The consensus sequences were put through a BLAST search in the NCBI GenBank nucleotide database to search for the fungal sequences of closest relatives that have been deposited in the NCBI database. Dissanayake et al.'s study [19] was followed for the phylogenetic analyses. Voglmayr and Beenken's study [79] was used as a reference of the dataset. Both individual and combined ITS, LSU, rpp2, and $t u b 2$ nucleotide sequences were analyzed. A total of 151 taxa were used for the phylogenetic analyses in order to find the taxonomic placement of each species. Three genera viz. Delonicicola, Furfurella (Delonicicolaceae), and Leptosillia (Leptosilliaceae) in Delonicicolales were used as the outgroup taxa.

The MAFFT online program was used to obtain initial alignments for each locus [94]. Alignments were manually edited and single gene sequence data sets were combined using MEGA7 [95]. The Alignment Transformation Environment online program was used to convert the file format [96]. MrModeltest [97] was used to find the best model for maximum likelihood (ML) and Bayesian analyses (BYPP). The six simultaneous Markov chains were run for 20,000,000 generations and trees were sampled every 1000th generation. Bayesian posterior probabilities from $\mathrm{MCMC}$ were evaluated with a final average standard deviation of the split frequency of $<0.01$. Bootstrap values for ML equal to or greater than $50 \%$ and BYPP equal to or greater than 0.90 are given at the nodes (Figure 1). Fig Tree v1.4.0 was used to configure the phylogenetic trees [98] and edited using Microsoft Office PowerPoint 2010 and Adobe Photoshop CS6 (Adobe Systems Incorporated, 345 Park Avenue, San Jose, CA, USA). 


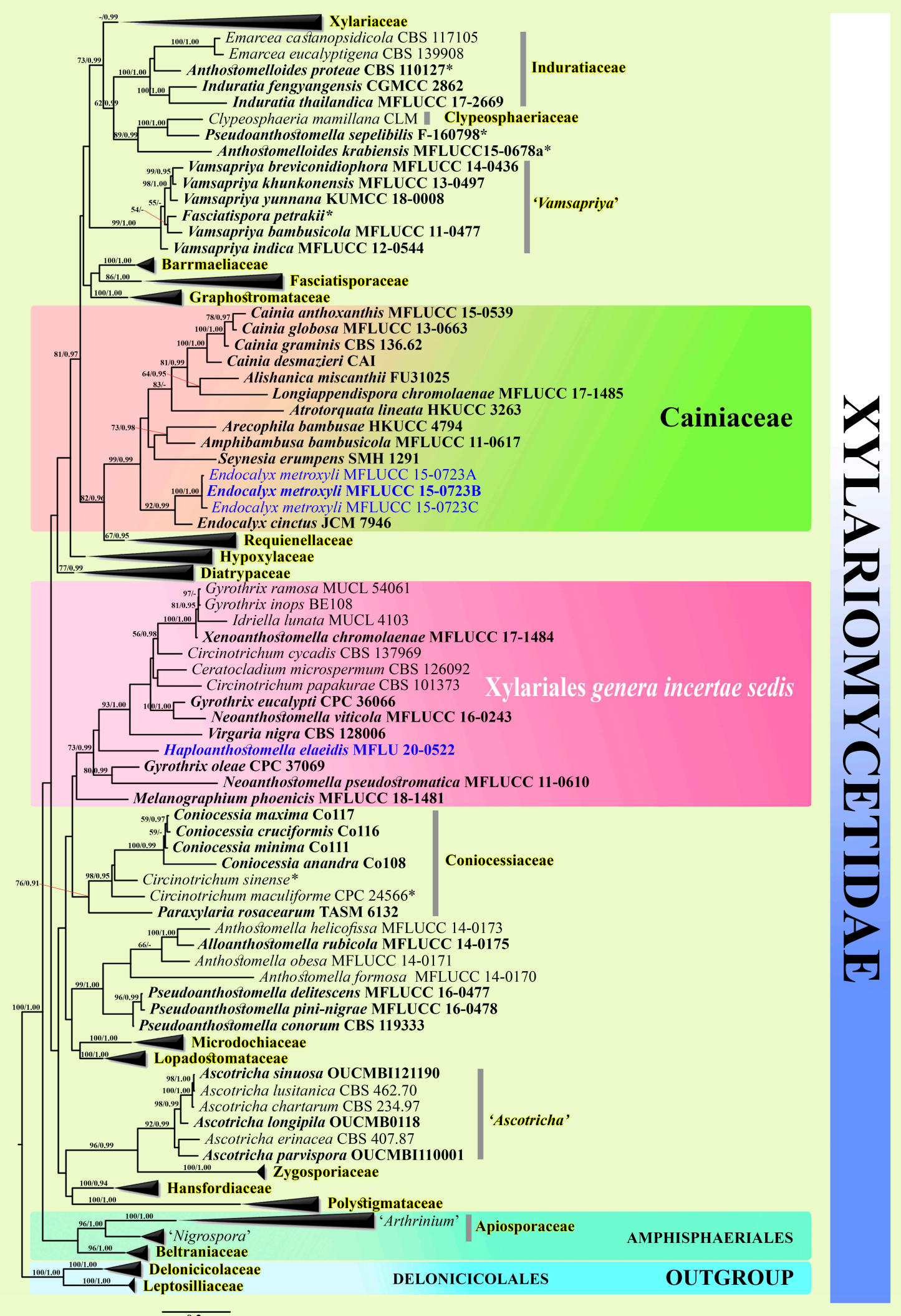

Figure 1. Maximum likelihood majority rule consensus tree for the analyses of selected Xylariomycetidae isolates based on a dataset of combined ITS, LSU, rpb2, and tub2 nucleotide sequence. Bootstrap support values for maximum likelihood (ML) equal to or higher than 50\% are given above each branch. Bayesian posterior probabilities (BYPP) equal to or greater than 0.90 are given at the nodes. Novel taxa are in blue bold and ex-type strains are in black bold. The tree is rooted to Delonicicolaceae and Leptosilliaceae (Delonicicolales). The asterisks represent unstable species. 


\section{Results}

\subsection{Morphology and Phylogeny}

The combined dataset comprised 151 taxa from selected taxa in Amphisphaeriales, Delonicicolales, and Xylariales (Table 1). The RAxML analyses of the combined dataset yielded the best-scoring tree (Figure 1) with a final ML optimization likelihood value of -126584.196783 . The matrix had 4598 distinct alignment patterns, with $65.07 \%$ undetermined characters or gaps. Estimated base frequencies were: $A=0.243574, C=0.257762$, $\mathrm{G}=0.258457, \mathrm{~T}=0.240207$; substitution rates $\mathrm{AC}=1.296272, \mathrm{AG}=3.089851, \mathrm{AT}=1.400263$, $\mathrm{CG}=1.060328, \mathrm{CT}=9.900102, \mathrm{GT}=1.000000$; gamma distribution shape parameter $\alpha$ $=0.443932$. Tree-Length $=25.372161$. Bayesian analysis resulted in a tree with similar topology and clades as the ML tree. Phylogenetic analyses of the combined ITS, LSU, rpb2, and $t u b 2$ loci show two novel taxa within the monospecific genus Haploanthostomella (type species Haploanthostomella elaeidis; Xylariales incertae sedis) and the novel taxa Endocalyx metroxyli, with the genus Endocalyx being placed in Cainiaceae.

\subsubsection{Haploanthostomella Konta \& K.D. Hyde. gen. nov.}

Index Fungorum number: IF557876; Facesoffungi number: FoF09173

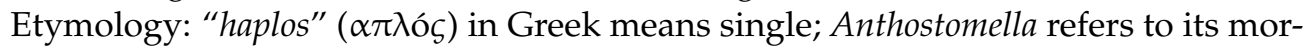
phological similarity to Anthostomella.

Saprobic on dead leaves and rachis in terrestrial habitats. Sexual morph: Ascomata immersed in the host epidermis, beneath a clypeus, visible as slightly raised blackened areas, dark brown to black, coriaceous, solitary or aggregated into clusters, scattered, with an ostiolar canal. Peridial wall thick, comprised of several layers of cells, outwardly comprising dark brown cells of textura prismatica and inwardly comprising hyaline cells of textura angularis. Paraphyses septate, tapering hyphae-like, hyaline. Asci eight-spored, unitunicate, clavate to cylindric, short pedicellate, with J-, apical ring. Ascospores unibiseriate into the asci, unicellular, obovoid, fusoid, hyaline or brown to dark brown, verrucose with a mucilaginous cap at apex. Germ slit straight, less than spore-length. Asexual morph: Not observed.

Type species: Haploanthostomella elaeidis Konta \& K.D. Hyde.

Notes: Anthostomella species were proven to be polyphyletic, and it is of no surprise that a new genus with anthostomella-like characteristics was discovered in this study [99]. Phylogenetic analyses based on a single dataset of ITS (supporting information section) and combined sequence data indicated that Haploanthostomella belongs to Xylariales genera incertae sedis, separating well from other genera but with low bootstrap values (Figure 1). According to the phylogenetic tree (Figure 1), seven genera (Ceratocladium, Circinotrichum, Gyrothrix, Idriella, Neoanthostomella, Virgaria and Xenoanthostomella) are closely related to our new genus, but morphological characteristics of these genera are different. The genera Neoanthostomella, Virgaria, and Xenoanthostomella were compared morphologically since they are similar to our new taxon. Haploanthostomella differs from Virgaria, Neoanthostomella, and Xenoanthostomella in having a J-apical ring, fusoid-obovoid ascospores, and verrucose with a mucilaginous cap at the apex, while Virgaria has asci with a J+ apical ring and smooth-walled elliposidal ascospores lacking of a mucilaginous sheath; Neoanthostomella smooth-walled elliposidal ascospores surrounded by a thick mucilaginous sheath; Xenoanthostomella has unilocular ascoma, and ascospores lacking germ slits and mucilaginous sheaths $[13,72,89]$. Therefore, Haploanthostomella is described here as a new genus based on phylogeny coupled with morphology. In addition, we provide a key to genera with Anthostomella-like characteristics.

\subsubsection{Haploanthostomella elaeidis Konta \& K.D. Hyde., sp. nov.} Index Fungorum number: IF557877, Facesoffungi number: FoF09174 (Figure 2) 

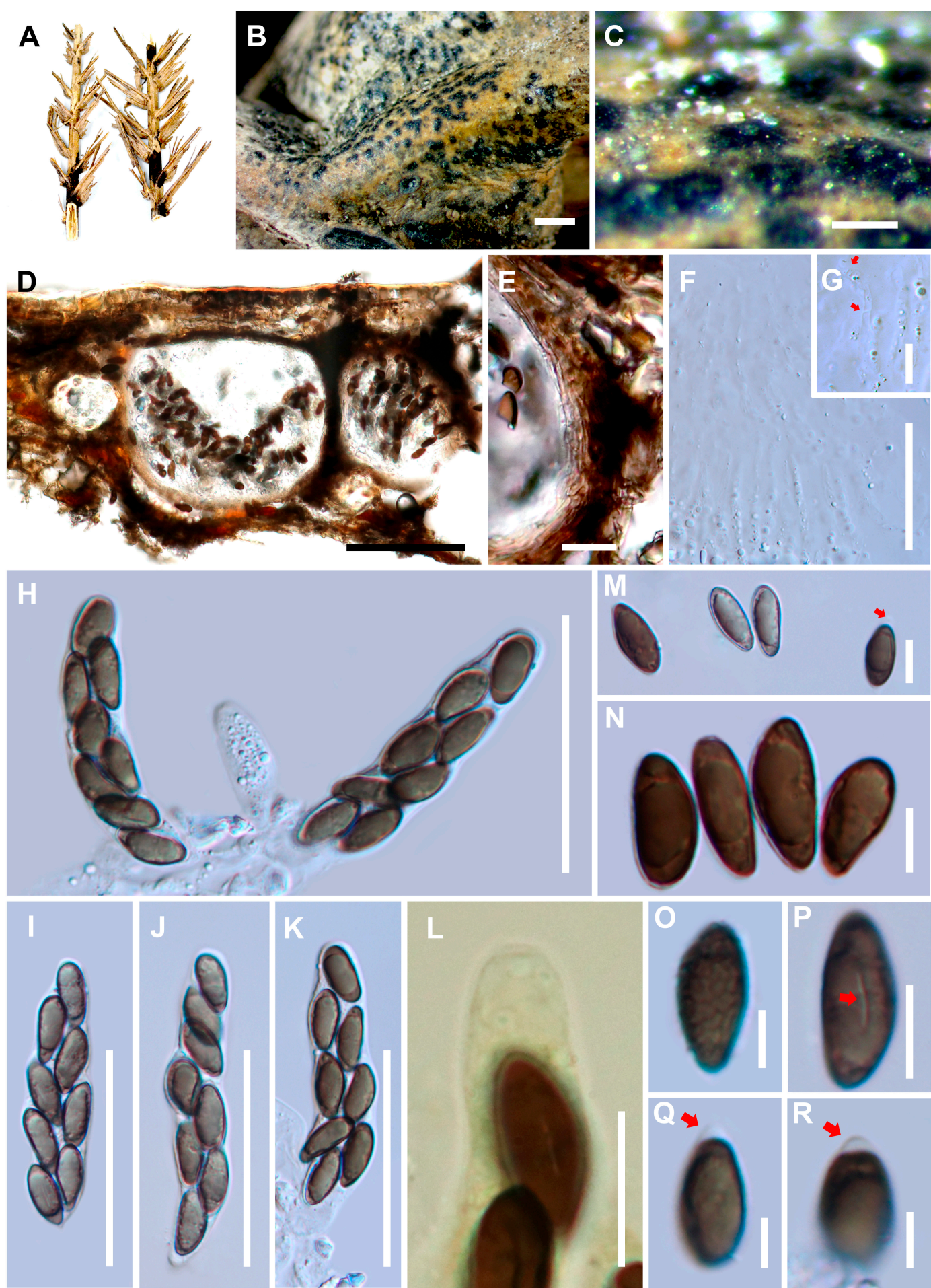

Figure 2. Haploanthostomella elaeidis (MFLU 20-0522, holotype). (A) Substrate. (B,C) Appearance of ascomata on the host surface. (D) Sections of ascomata. (E) Peridium. (F) Hamathecium. (G) Septa of paraphyses show in red arrows. (H,I-K) Asci. (L) J- apical ring in Melzer's reagent. (M,N,P-R) Ascospores with mucilaginous cap (red arrows in M, Q, R) and germ slit (red arrows in P). (O) An ascospore with verrucose wall. Scale bars: $B=1000 \mu \mathrm{m}, C=200 \mu \mathrm{m}, \mathrm{D}=500 \mu \mathrm{m}, \mathrm{E}$, $\mathrm{G}, \mathrm{L}=20 \mu \mathrm{m}, \mathrm{F}, \mathrm{H}-\mathrm{K}=50 \mu \mathrm{m}, \mathrm{M}-\mathrm{P}=10 \mu \mathrm{m}, \mathrm{Q}-\mathrm{R}=5 \mu \mathrm{m}$.

Etymology: Referring to the genus of palm trees Elaeis Jacq.

Holotype: MFLU 20-0522.

Saprobic on dead leaves and rachis of Elaeis guineensis. Sexual morph: Ascomata $160-280 \times 130-350 \mu \mathrm{m}\left(x^{-}=220 \times 240 \mu \mathrm{m}, \mathrm{n}=20\right)$, immersed in the host 
epidermis, beneath a clypeus, visible as slightly raised blackened areas, dark brown to black, coriaceous, solitary or aggregated into clusters, scattered, with an ostiolar canal. Peridial wall 13-45 $\mu \mathrm{m}$ wide, thick, comprising several layers of cells, outwardly comprising dark brown cells of textura irregularis and inwardly comprising hyaline cells of textura prismatica, 7-20 $\mu \mathrm{m}$ wide. Paraphyses $1.5-4.5 \mu \mathrm{m}$ wide, septate, hyphae-like, hyaline. Asci 50-90 $\times 10-15 \mu \mathrm{m}\left(x^{-}=70 \times 12 \mu \mathrm{m}, \mathrm{n}=40\right), 8$-spored, unitunicate, clavate to cylindric, short pedicellate, with J- apical ring. Ascospores 10-18 $\times 5-8 \mu \mathrm{m}\left(x^{-}=14 \times 6 \mu \mathrm{m}, \mathrm{n}=100\right)$, uni-biseriate into the asci, unicellular, obovoid, fusoid, hyaline to light brown when immature and brown to dark brown when mature, mostly one, rarely two-guttulate, cell wall verrucose, with a mucilaginous cap at the apex. Germslit 3-6 $\mu \mathrm{m}$ length $\left(x^{-}=5 \mu \mathrm{m}, \mathrm{n}=50\right)$, straight, less than spore-length. Asexual morph: Not observed.

Material examined: THAILAND, Surat Thani Province, on dead leaves and rachis of Elaeis guineensis Jacq. (Arecaceae) on the ground, 21 July 2017, Sirinapa Konta, SRWD12 (MFLU 20-0522, holotype).

Notes: A BLAST search of $H$. elaeidis ITS sequence shows $83.87 \%$ similarity with Gyrothrix oleae (CPC 37069); LSU sequence shows 95.95\% similarity with Gyrothrix eucalypti (CPC 36066); and rpb2 sequence shows 80.95\% similarity with Lopadostoma meridionale (LG). Only the sexual morph of $H$. elaeidis was found in nature, and we could not obtain a pure culture from fresh samples. Therefore, the morphological characteristics of $H$. elaeidis were not compared with Ceratocladium, Circinotrichum, Gyrothrix, and Idriella, as they only had asexual morphs found in nature. Hence, the morphological features of H. elaeidis were only compared with Neoanthostomella, Virgaria, and Xenoanthostomella, as they have sexual morphs.
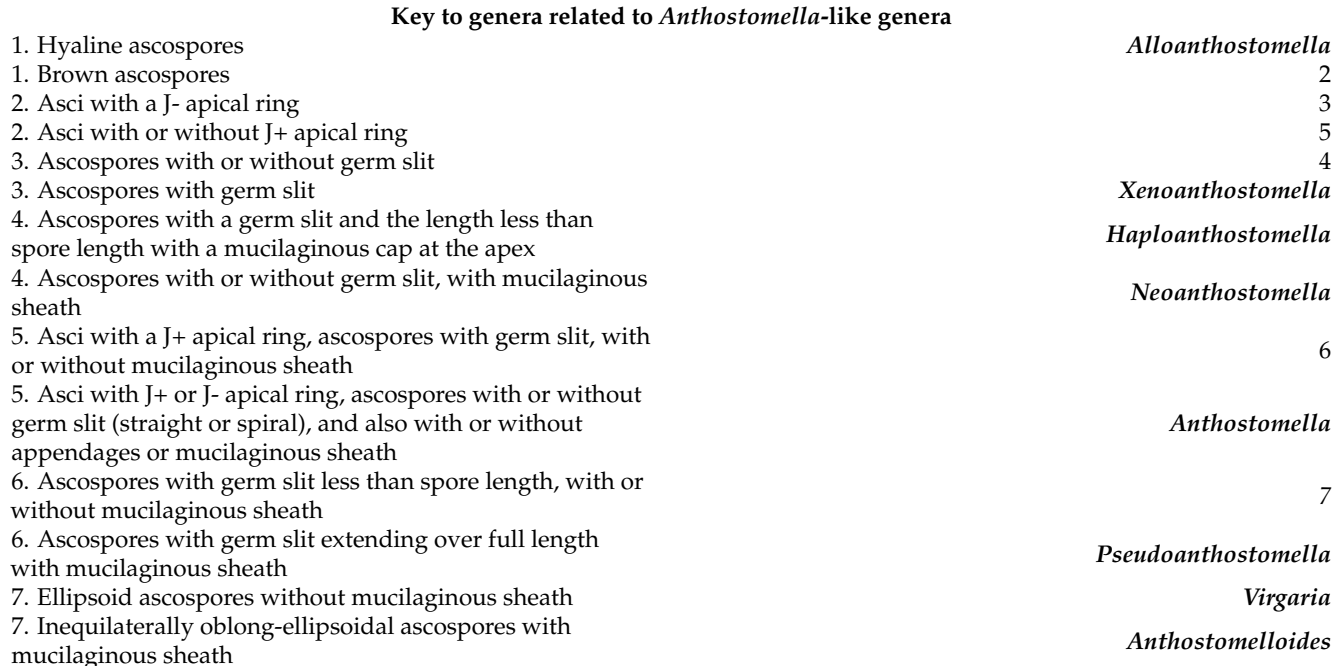

3.1.3. Endocalyx Berk. \& Broome, J. Linn. Soc., Bot. 15(1): 84 (1876) [1877]

Index Fungorum number: IF8158; Facesoffungi number: FoF09175

Saprobic on various plants. Colonies on host plant, pustules nearly flat or raised, circular, discolored, dark brown to black, at last bursting, the conidiomata developing. Sexual morph: Undetermined. Asexual morph: Conidiomata scattered, erect, cupulate to cylindrical; peridial hyphae enclosing the inner conidial mass, nonsporiferous, brown to yellowish brown; some species consisting of two parts of conidioma: (1) a basal cylinder covering a central column, rough-walled, carbonaceous, composed of black hyphae which are sometimes branched and are adherent to one another; (2) a slender central column, synnematous, expanding radially apically, high, enclosed by the peridial hyphae which are nonsporiferous, orange-yellow to lemon-yellow. Peridial wall thick, comprising dark brown, thick-walled cells of textura angularis. Conidiophores thread-like, septate, with or without short pegs bearing the conidia, meristematic at the base, colorless basally and gradually turning brown apically, 1-2 $\mu \mathrm{m}$ wide; peridium thick, comprising dark brown, thick-walled cells of textura angularis. Conidiogenous cells holoblastic, integrated, determinate. Conidia 
solitary, unicellular, flattened, round, oval or slightly polygonal in face view, at first pale, dark brown to fuscous black at maturity, with or without guttules, often with a longitudinal hyaline straight germ slit extending the full-length (adapted from [99-101]).

Type species: Endocalyx thwaitesii Berk. \& Broome

Notes: Endocalyx is a coelomycetous genus in Cainiaceae with E. cinctus collected from Japan E. metroxyli sp. nov. collected from Thailand. Phylogenetic analyses of a single dataset of ITS (supporting information section) and phylogenetic analyses of a combined dataset of ITS, LSU, rpb2, and tub2 regions (Figure 1) confirm the placement of Endocalyx within Cainiaceae. ITS analyses showed that Endocalyx is closely related to Amphibambusa and Atrotorquata (supporting information section), while Figure 1 shows that Endocalyx formed a basal clade to other cainiaceous genera (Alishanica, Amphibambusa, Arecophila, Atrotorquata, Cainia, Longiappendispora, and Seynesia) with high bootstrap support. Morphologically, Endocalyx has been revised and described only as an asexual morph of the genus $[100,101]$, while all genera in Cainiaceae have been described in their sexual morphs, except the type genus Cainia, for which both asexual and sexual morphs have been described. We could not compare the morphology of Endocalyx to Arecophila, Seynesia, and Amphibambusa (sister species in Figure 1). Therefore, Cainia was used for morphological comparisons; Endocalyx differs from Cainia in having erect conidiomata and also the ostiole opening surrounded by yellow hyphae, ellipsoid-globose conidia, unicellular with brown to dark brown color, and a germ slit. Cainia has immersed conidiomata, conidiogenous cells with one to three phialides, and elongate fusiform conidia, unicellular or septate, hyaline, with pointed ends [100-102].

Table 2. Host and locality information of Endocalyx reported worldwide based on the records of Species Fungorum 2021.

\begin{tabular}{|c|c|c|c|c|c|}
\hline \multirow{2}{*}{ No. } & \multirow{2}{*}{ Species } & \multicolumn{2}{|r|}{ Host } & \multirow{2}{*}{ Country } & \multirow{2}{*}{ Reference } \\
\hline & & Eudicots & Monocots & & \\
\hline 1 & $\begin{array}{c}\text { Endocalyx } \\
\text { amarkantakensis }\end{array}$ & $\begin{array}{c}\text { Shorea robusta } \\
\text { (Dipterocarpaceae) }\end{array}$ & & India (Holotype) & [103] \\
\hline \multirow{6}{*}{2} & \multirow{6}{*}{ E. cinctus* } & & $\begin{array}{l}\text { Livistona chinensis var. boninensis } \\
\text { (Arecaceae; solitary palm) }\end{array}$ & Japan & [104] \\
\hline & & & $\begin{array}{c}\text { Oncosperma fasciculatum } \\
\text { (Arecaceae; clustering, rarely } \\
\text { solitary palm) }\end{array}$ & Japan & [101] \\
\hline & & & $\begin{array}{c}\text { Oncosperma sp. (Arecaceae; } \\
\text { clustering, rarely solitary palm) }\end{array}$ & $\begin{array}{l}\text { Sri Lanka } \\
\text { (Holotype) }\end{array}$ & [100] \\
\hline & & & $\begin{array}{c}\text { Phoenix canariensis (Arecaceae; } \\
\text { solitary palm) }\end{array}$ & Japan & [101] \\
\hline & & & $\begin{array}{c}\text { Phoenix hanceana (Arecaceae; } \\
\text { solitary palm) }\end{array}$ & Hong Kong & [105] \\
\hline & & & $\begin{array}{l}\text { Trachycarpus fortunei (Arecaceae; } \\
\text { solitary palm) }\end{array}$ & Japan & [101] \\
\hline 3 & E. collantesis & & Smilax sp. (Smilacaceae) & Cuba (Holotype) & [106] \\
\hline 4 & E. indicus & twigs of woody & & India (Holotype) & [107] \\
\hline \multirow{2}{*}{5} & \multirow{2}{*}{ E. indumentum } & & $\begin{array}{l}\text { Livistona chinensis var. boninensis } \\
\text { (Arecaceae; solitary palm) }\end{array}$ & Japan (Holotype) & {$[101,104]$} \\
\hline & & & $\begin{array}{c}\text { Phoenix canariensis (Arecaceae; } \\
\text { solitary palm) }\end{array}$ & Japan & [104] \\
\hline
\end{tabular}


Table 2. Cont.

\begin{tabular}{|c|c|c|c|c|c|}
\hline \multirow{2}{*}{ No. } & \multirow{2}{*}{ Species } & \multicolumn{2}{|r|}{ Host } & \multirow{2}{*}{ Country } & \multirow{2}{*}{ Reference } \\
\hline & & Eudicots & Monocots & & \\
\hline & & & Acrocomia mexicana (Arecaceae) & Mexico & [108] \\
\hline \multirow{29}{*}{6} & \multirow{29}{*}{ E. melanoxanthus } & & \multirow{4}{*}{$\begin{array}{l}\text { Archontophoenix alexandrae } \\
\text { (Arecaceae; solitary palm) }\end{array}$} & Australia & [109] \\
\hline & & & & Hong Kong & {$[105,109]$} \\
\hline & & & & Malaysia & [109] \\
\hline & & & & Singapore & [109] \\
\hline & & & Arecaceae & Mexico & [108] \\
\hline & & & Arenga engleri (Arecaceae; & Hong Kong & [105] \\
\hline & & & clustering palm) & Japan & [104] \\
\hline & & & $\begin{array}{c}\text { Dypsis lutescens } \\
\text { (=Chrysalidocarpus lutescens) } \\
\text { (Arecaceae; clustering palm) }\end{array}$ & Japan & [104] \\
\hline & & & $\begin{array}{c}\text { Caryota urens (Arecaceae; } \\
\text { solitary palm) }\end{array}$ & $\begin{array}{l}\text { Sri Lanka } \\
\text { (Holotype) }\end{array}$ & [100] \\
\hline & & & \multirow{8}{*}{$\begin{array}{c}\text { Cocos nucifera (Arecaceae; } \\
\text { solitary palm) }\end{array}$} & Australia & [109] \\
\hline & & & & Ghana & [110] \\
\hline & & & & Hawaii & {$[111,112]$} \\
\hline & & & & Japan & [104] \\
\hline & & & & Malaysia & {$[109,113]$} \\
\hline & & & & $\begin{array}{l}\text { Papua New } \\
\text { Guinea }\end{array}$ & [114] \\
\hline & & & & Seychelles & [109] \\
\hline & & & & Singapore & [109] \\
\hline & & $\begin{array}{l}\text { Coffea arabica } \\
\text { (Rubiaceae) }\end{array}$ & & Venezuela & [115] \\
\hline & & & \multirow{2}{*}{$\begin{array}{c}\text { Dracaena fragrans } \\
\text { (Asparagaceae) }\end{array}$} & Cuba & [116] \\
\hline & & & & Venezuela & [115] \\
\hline & & & \multirow{3}{*}{$\begin{array}{c}\text { Elaeis guineensis (Arecaceae; } \\
\text { solitary palm) }\end{array}$} & Ghana & [110] \\
\hline & & & & Myanmar & [117] \\
\hline & & & & Sierra Leone & [113] \\
\hline & & & $\begin{array}{l}\text { Elaeis sp. (Arecaceae; solitary } \\
\text { palm) }\end{array}$ & Japan & [104] \\
\hline & & & $\begin{array}{c}\text { Licuala longicalycata (Arecaceae; } \\
\text { solitary palm) }\end{array}$ & Thailand & [118] \\
\hline & & & $\begin{array}{c}\text { Livistona chinensis (Arecaceae; } \\
\text { solitary palm) }\end{array}$ & Hong Kong & [105] \\
\hline & & & $\begin{array}{l}\text { Livistona chinensis var. boninensis } \\
\text { (Arecaceae; solitary palm) }\end{array}$ & Japan & [104] \\
\hline & & & $\begin{array}{l}\text { Livistona rotundifolia (Arecaceae; } \\
\text { solitary palm) }\end{array}$ & Taiwan & [119] \\
\hline & & & $\begin{array}{c}\text { Livistona speciosa (Arecaceae; } \\
\text { solitary palm) }\end{array}$ & Myanmar & [117] \\
\hline
\end{tabular}


Table 2. Cont.

\begin{tabular}{|c|c|c|c|c|c|}
\hline \multirow{2}{*}{ No. } & \multirow{2}{*}{ Species } & \multicolumn{2}{|r|}{ Host } & \multirow{2}{*}{ Country } & \multirow{2}{*}{ Reference } \\
\hline & & Eudicots & Monocots & & \\
\hline & & & $\begin{array}{c}\text { Nannorrhops ritchieana } \\
\text { (Arecaceae; clustering palm) }\end{array}$ & Pakistan & {$[120]$} \\
\hline & & & $\begin{array}{c}\text { Phoenix canariensis (Arecaceae; } \\
\text { solitary palm) }\end{array}$ & Japan & {$[104]$} \\
\hline & & & $\begin{array}{c}\text { Phoenix hanceana (Arecaceae; } \\
\text { solitary palm) }\end{array}$ & Hong Kong & {$[105,121]$} \\
\hline & & & $\begin{array}{c}\text { Phoenix reclinata (Arecaceae; } \\
\text { solitary palm) }\end{array}$ & Ghana & {$[110]$} \\
\hline & & & $\begin{array}{c}\text { Phoenix roebelenii (Arecaceae; } \\
\text { solitary palm) }\end{array}$ & Japan & {$[104]$} \\
\hline & & & $\begin{array}{l}\text { Phoenix roebelenii-senegalensis } \\
\text { (Arecaceae; solitary palm) }\end{array}$ & Japan & {$[104]$} \\
\hline & & & Ravenala madagascariensis & Japan & {$[104]$} \\
\hline & & & (Strelitziaceae) & Taiwan & {$[119]$} \\
\hline & & & $\begin{array}{l}\text { Ripogonum scandens } \\
\text { (Ripogonaceae) }\end{array}$ & New Zealand & {$[122]$} \\
\hline & & & $\begin{array}{l}\text { Roystonea borinquena (Arecaceae; } \\
\text { solitary palm) }\end{array}$ & USA (Florida) & {$[123]$} \\
\hline & & & $\begin{array}{c}\text { Roystonea regia (Arecaceae; } \\
\text { solitary palm) }\end{array}$ & Cuba & [124-127] \\
\hline & & & $\begin{array}{l}\text { Sabal palmetto (Arecaceae; } \\
\text { solitary palm) }\end{array}$ & USA (Florida) & {$[128]$} \\
\hline & & & $\begin{array}{l}\text { Serenoa serrulata (Arecaceae; } \\
\text { clustering and solitary palm) }\end{array}$ & USA (Florida) & {$[129]$} \\
\hline & & & Smilax sp. (Smilacaceae) & USA (Florida) & {$[128]$} \\
\hline & & & $\begin{array}{l}\text { Trachycarpus fortunei (Arecaceae; } \\
\text { solitary palm) }\end{array}$ & China & [109] \\
\hline & & & & Australia & {$[109]$} \\
\hline & & & & China & [109] \\
\hline & & & unknown palm & Hong Kong & [109] \\
\hline & & & & Malaysia & [109] \\
\hline & & & & Seychelles & [109] \\
\hline & & & & Singapore & [109] \\
\hline & & & $\begin{array}{l}\text { Wodyetia bifurcata (Arecaceae; } \\
\text { solitary palm) }\end{array}$ & Florida & [123] \\
\hline & $\begin{array}{c}\text { E. melanoxanthus } \\
(=\text { E. melanoxanthus } \\
\text { var. grossus })\end{array}$ & & $\begin{array}{l}\text { Trachycarpus fortunei (Arecaceae; } \\
\text { solitary palm) }\end{array}$ & Japan & [101] \\
\hline
\end{tabular}


Table 2. Cont

\begin{tabular}{|c|c|c|c|c|c|}
\hline \multirow{2}{*}{ No. } & \multirow{2}{*}{ Species } & \multicolumn{2}{|r|}{ Host } & \multirow{2}{*}{ Country } & \multirow{2}{*}{ Reference } \\
\hline & & Eudicots & Monocots & & \\
\hline & \multirow{15}{*}{$\begin{array}{l}\text { E. melanoxanthus } \\
(=\text { E. melanoxanthus } \\
\text { var. melanoxanthus })\end{array}$} & & $\begin{array}{c}\text { Acrocomia intumescens } \\
\text { (Arecaceae; solitary palm) }\end{array}$ & Brazil & [102] \\
\hline & & & $\begin{array}{l}\text { Butia yatay (Arecaceae; solitary } \\
\text { palm) }\end{array}$ & Argentina & [130] \\
\hline & & & $\begin{array}{c}\text { Cocos nucifera (Arecaceae; } \\
\text { solitary palm) }\end{array}$ & Ghana & [101] \\
\hline & & & $\begin{array}{l}\text { Euterpe edulis (Arecaceae; } \\
\text { solitary, or rarely clustering } \\
\text { palm (growing in dense tufts or }\end{array}$ & Argentina & [130] \\
\hline & & & stems) & Brazil & [102] \\
\hline & & & $\begin{array}{l}\text { Euterpe oleracea (Arecaceae; } \\
\text { clustering palm) }\end{array}$ & Brazil & [102] \\
\hline & & & $\begin{array}{l}\text { Livistona chinensis var. boninensis } \\
\text { (Arecaceae; solitary palm) }\end{array}$ & Japan & [101] \\
\hline & & & $\begin{array}{l}\text { Livistona chinensis var. subglobosa } \\
\text { (Arecaceae; solitary palm) }\end{array}$ & Japan & [101] \\
\hline & & & $\begin{array}{c}\text { Phoenix canariensis (Arecaceae; } \\
\text { solitary palm) }\end{array}$ & Japan & [101] \\
\hline & & & $\begin{array}{c}\text { Phoenix roebelenii (Arecaceae; } \\
\text { solitary palm) }\end{array}$ & Japan & [101] \\
\hline & & & $\begin{array}{l}\text { Satakentia liukiuensis (Arecaceae; } \\
\text { solitary palm) }\end{array}$ & Japan & [101] \\
\hline & & & $\begin{array}{c}\text { Syagrus coronata (Arecaceae; } \\
\text { solitary palm) }\end{array}$ & Brazil & [131] \\
\hline & & & $\begin{array}{c}\text { Syagrus romanzoffiana } \\
\text { (Arecaceae; solitary palm) }\end{array}$ & Argentina & [130] \\
\hline & & & $\begin{array}{l}\text { Trachycarpus fortunei (Arecaceae; } \\
\text { solitary palm) }\end{array}$ & Japan & [101] \\
\hline & & & $\begin{array}{l}\text { Washingtonia robusta (Arecaceae; } \\
\text { solitary palm) }\end{array}$ & Japan & [101] \\
\hline \multirow{5}{*}{7} & \multirow{5}{*}{$\begin{array}{c}\text { E. thwaitesii (Type } \\
\text { species) }\end{array}$} & $\begin{array}{l}\text { Cissus oreophila } \\
\text { (Vitaceae) }\end{array}$ & & Ghana & [132] \\
\hline & & \multirow{4}{*}{$\begin{array}{l}\text { Cissus sp. } \\
\text { (Vitaceae) }\end{array}$} & & Ghana & [133] \\
\hline & & & & Sri Lanka & [133] \\
\hline & & & & Ghana & [133] \\
\hline & & & clustering, rarely solitary palm) & $\begin{array}{l}\text { Sri Lanka } \\
\text { (Holotype) }\end{array}$ & [133] \\
\hline
\end{tabular}

* Have molecular data. 

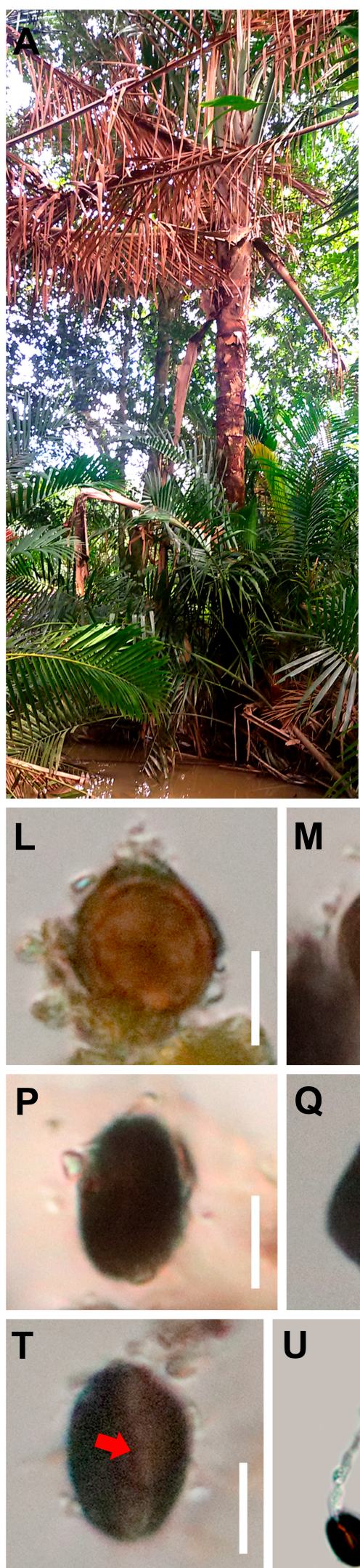
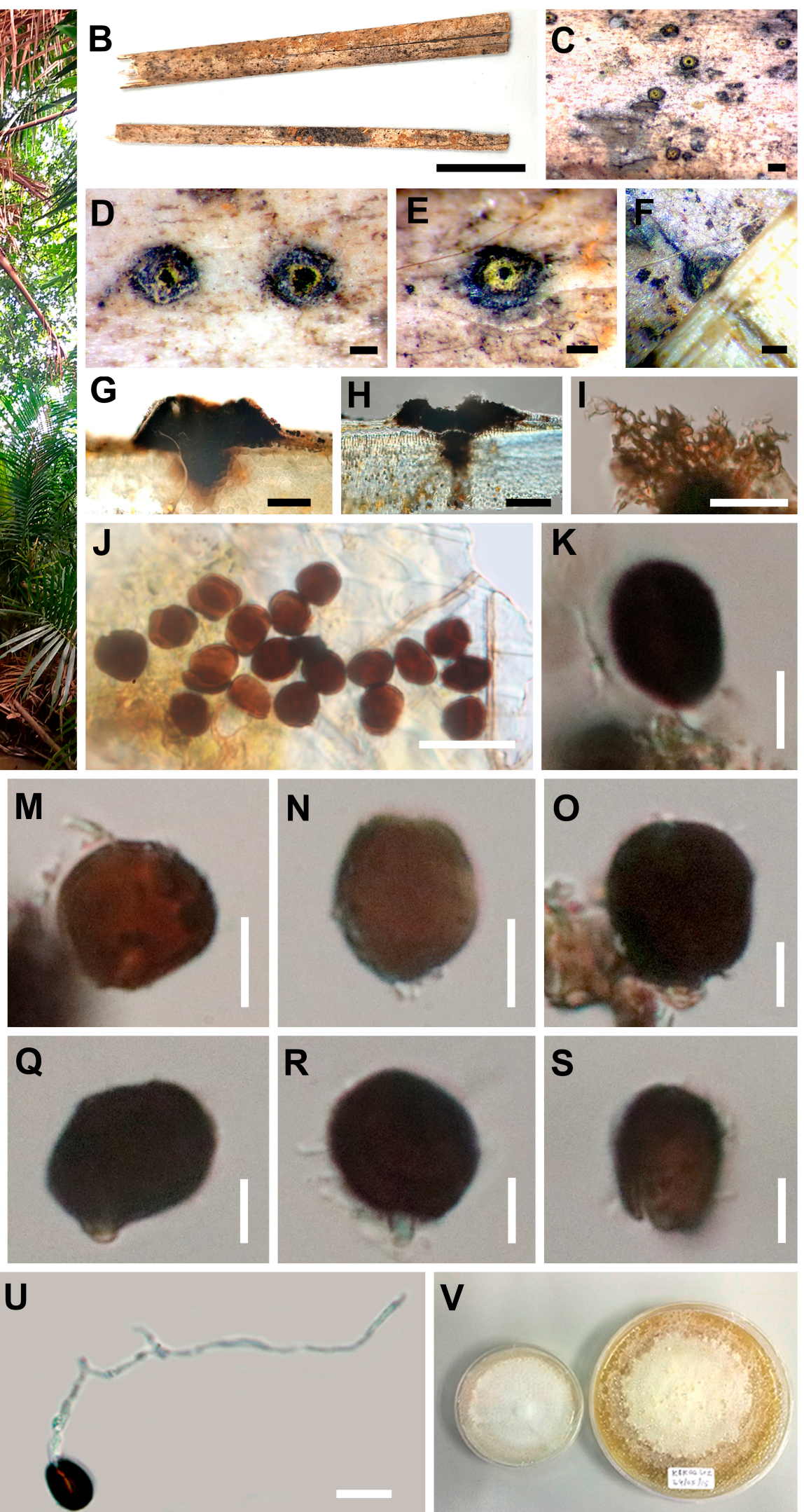

Figure 3. Endocalyx metroxyli (MFLU 15-1454, holotype). (A) Forest in Krabi Province. (B) Palm samples. (C-E) Appearance of conidiomata on host. (F) Vertical cut of a conidioma. (G-H) Vertical section of a conidioma. (I) Section of peridium. (J) Group of conidia. (K) Conidiophores reduced to conidiogenous cell with conidium. (L-S) Conidia (P-R, Conidia with conidiogenous cells). (T) Germ slit (red arrow). (U) Germinated conidia. (V) Colonies on MEA media. Scale bars: B $=2 \mathrm{~cm}$, $\mathrm{C}=500 \mu \mathrm{m}, \mathrm{D}-\mathrm{H}=200 \mu \mathrm{m}, \mathrm{I}, \mathrm{J}=20 \mu \mathrm{m}, \mathrm{L}-\mathrm{T}=5 \mu \mathrm{m}, \mathrm{U}=10 \mu \mathrm{m}$. 
Recently, Longiappendispora was introduced under Cainiaceae, with seven genera in total included in the family by Mapook et al. [11]. In our study, detailed molecular analyses were done for Endocalyx and its placement in Cainiaceae (Xyalriales) was confirmed. Previously, Endocalyx was classified in Apiosporaceae (Xylariales, Sordariomycetes) based on morphological evidence. As the first detailed molecular data of Endocalyx cinctus have been made available from a Japan laboratory [32], their current placement is supported (Figure 1). However, there are no recent publications referring to the molecular data of this genus yet. Thus, in this study, we present the placement of Endocalyx based on multigene phylogenetic analyses with recent sequence data from the Japan collection as well as the Thailand collection. In addition, we accept eight genera in Cainiaceae (Alishanica, Amphibambusa, Arecophila, Atrotorquata, Cainia, Endocalyx, Longiappendispora, and Seynesia), and seven species by including our new species in the genus Endocalyx (Table 2). In addition, we provide a key for the members of Cainiaceae.

\subsubsection{Endocalyx metroxyli Konta \& K.D. Hyde. sp. nov.}

Index Fungorum number: IF558116, Facesoffungi number: FoF09176 (Figure 3)

Etymology: Refers to the name of the host genus, Metroxylon.

Holotype: MFLU 15-1454.

Saprobic on dead petiole of Metroxylon sagu. Colonies on host plant, pustules. Sexual morph: Undetermined. Asexual morph: Conidiomata 340-660 $\mu \mathrm{m}$ wide, in vertical section 495-820 × 325-485 $\mu \mathrm{m}$, acervulus, solitary, semi-immersed to immersed in the host epidermis, beneath a clypeus, visible as slightly raised and blackened, black, carbonaceous, fragile, with an ostiolar canal. Ostiolar opening surrounded by a yellow margin. Peridial wall 34-80 $\mu \mathrm{m}$ wide, thick, comprising dark brown cells of textura angularis. Conidiomata not observed with a basal cylinder covering a central column or a slender central column in our collection. Conidiophores reduced to conidiogenous cell, hyaline to pale-brown, unbranched, smooth. Conidia 13-16 × 7-10 $\mu \mathrm{m}\left(x^{-}=13 \times 10 \mu \mathrm{m}, \mathrm{n}=30\right)$, unicellular, ellipsoid-globose, brown to dark brown, with short pegs bearing conidia, with germ slit, smooth-walled.

Culture characteristics: Colonies on MEA, at first white, raised, effuse, velvety to hairy, circular, smooth at the margin, white from above, pale-brown from below.

Material examined: Thailand, Krabi Province, on dead petiole of Metroxylon sagu Rottb. on the ground (Arecaceae), 8 December 2014, Sirinapa Konta KBR04h2 (MFLU 15-1454, holotype); ex-type living culture, MFLUCC 15-0723A; ibid. MFLUCC 15-0723B, MFLUCC 15-0723C.

Additional sequence data: SSU: MT929310, MT929311, tef1- $\alpha$ : MT928152, MT928153.

Notes: Endocalyx metroxyli is phylogenetically well supported and is placed in Cainiaceae (Figure 1). Endocalyx metroxyli is closely related to E. cinctus with high bootstrap support but is distinct in morphological characteristics. A BLAST search of E. metroxyli ITS sequence shows $83.10 \%$ similarity with Requienella seminuda (CBS 140502) (CPC 37069), LSU sequence shows $96.14 \%$ similarity with Entosordaria quercina (RQ), tub2 sequence shows $88.94 \%$ similarity with Daldinia dennisii var. dennisii, SSU sequence shows $97.92 \%$ similarity with Xenoanthostomella chromolaenae (MFLUCC 17-1484), and tef1- $\alpha$ sequence shows $89.39 \%$ similarity with Barrmaelia macrospor (BM).

Endocalyx metroxyli is morphologically similar to E. melanoxanthus. However, Endocalyx metroxyli does not have erect conidiomata developing from the pustules, as was mentioned by Petch [100], Okada and Tubaki [101], and Vitoria et al. [102,131]. In this study, we found only a black raised pustule structure with ostiole surrounded by a yellow hyphae ring, and hyaline conidiophore, unicellular, dark brown conidia with a longitudinal germ slit. Endocalyx melanoxanthus was collected and described from palm hosts (Arecaceae), and a few collections were collected from other host plants (Table 2). According to Species Fungorum [134], E. melanoxanthus var. Grossus (G. Okada \& Tubaki) and E. melanoxanthus var. melanoxanthus (Berk. \& Broome) are considered as E. melanoxanthus, even though they have several different characteristics. 
Endocalyx metroxyli is morphologically similar to E. melanoxanthus var. melanoxanthus, in having black raised pustules surrounded by yellow hyphae and smooth-walled conidia with no significant size differences [100-102]. However, our new taxon lacks cupulate or cylindrical conidiomata [101,102]. On the other hand, E. metroxyli differs from E. melanoxanthus var. grossus by lacking the production of ornamented conidia [100,101].

1. Asexual morph

Keys to genera of Cainiaceae

1.1 Coelomycetous; $1-3$ phialides conidiogenous cells, and elongate fusiform conidia with unicellular or septate, with Cainia pointed ends

1.1 Coelomycetous; conidiomata with ostiolar opening surrounded by yellow, with unicellular conidia, ellipsoid-globose, pale to dark brown to black, with a straight germ slit Endocalyx extending the full-length

\section{Sexual morph}

2.1 Cylindrical-clavate asci, ascospores with 1-septate

2.1 Cylindrical, or cylindrical to elongate cylindrical asci, ascospores with 1-septate 2.2 Ellipsoidal ascospores, with brown, and sheath 2.2 Ellipsoidal to fusiform ascospores, with brown, and sheath 2.3 Ellipsoid to broadly fusiform ascospores, longitudinal striations, bristle-like polar appendages from both ends, without a gelatinous sheath 2.3 Fusiform to broad-fusiform ascospores with pointed at both ends, striation wall, and sheath

2.3 Ellipsoidal or oblong ascospores

2.4 Oblong ascospores with cap-like appendage, germ slits

2.4 Ellipsoidal ascospores

2.5 Ascospores with striation wall, brown, and sheath

2.5 Ascospores with striate or verrucose wall, and subhyaline to brown

Cainia

Atrotorquata

Longiappendispora

Amphibambusa

Seynesia

Alishanica

Arecophila

\section{Discussion}

Based on phylogeny and morphological characteristics, the new monotypic genus Haploanthostomella (type species: Haploanthostomella elaeidis) and the new species Endocalyx metroxyli have been established. The former new species was isolated from a dead rachis of Elaeis guineensis, and the latter from a dead petiole of Metroxylon sagu (Arecaceae) in Thailand. Phylogenetic analyses of combined datasets together with morphological characteristics revealed that Haploanthostomella belongs to Xylariales incertae sedis, while Endocalyx belongs to the Cainiaceae (Xylariales).

Based on morphological features, Endocalyx was assigned to Apiosporaceae (Amphisphaeriales, Sordariomycetes), together with four other genera, viz. Appendicospora, Arthrinium, Dictyoarthrinium, and Nigrospora [3,8]. Later, Dictyoarthrinium was transferred to Didymosphaeriaceae (Pleosporales, Dothideomycetes) [135]. According to our phylogenetic analyses (Figure 1), Arthrinium and Nigrospora should be accepted under the Apiosporaceae, while Appendicospora did not clade to this family (supporting information section), and Endocalyx fits well within the Cainiaceae.

Interestingly, four out of seven species in the genus Endocalyx (E. melanoxanthus, E. cinctus, E. indumentum, and E. thwaitesii) were collected from palm hosts (Table 2). 
Endocalyx metroxyli is similar to other species by having dark brown conidia with a fulllength germ slit, it but differs from other species by not having conidiomata produced from the pustulate and no thread-like structure of conidiophores. Morphological characteristics of species in the genus are mostly flat or raised pustules, capsule or slender conidiomata with or without branches at the apex, and brown to dark brown conidia with smooth walls (E. amarkantakensis, E. collantesis, E. indumentum, E. melanoxanthus, E. melanoxanthus var. melanoxanthus), while some species are verrucose-walled (E. cinctus, E. indumentum, E. melanoxanthus var. grossus, E. thwaitesii). We referred to previous publications for morphological comparisons to the taxa in this study, as we did not observe all holotype specimens [100-102].

According to the literature, there are also strains derived from another two species and two varieties. Excluding E. cinctus, no sequence data are available for generic types of Endocalyx and other species, and their morphology and host substrates are closely related to our novel taxon. Endocalyx species have been reported in several countries, especially in tropical and subtropical regions. Furthermore, palm trees (Arecaceae) have most commonly been reported as the host, while several species have been presented from other hosts (Table 2).

The phylogenetic placement of many groups within the Xylariales remains unclear (e.g., Anthostomelloides, Calceomyces, Circinotrichum, Fasciatispora (only F. petrakii), Gyrothyrix, Melanographium, Neoanthostomella, Pseudoanthostomella, and Xenoanthostomella, Figure 1). Thus, it is necessary to collect and analyze more fungal specimens from Xylariales using multigene phylogeny (with protein coding genes) and morphology to resolve their taxonomical placement and delimitation.

Author Contributions: Conceptualization, S.K.; Formal analysis, S.K.; Funding acquisition, K.D.H. and S.T.; Methodology, S.K.; Resources, S.C.K., J.X. and S.T.; Supervision, K.D.H. and P.D.E.; Writingoriginal draft, S.K., S.C.K., M.C.S., S.T.A., L.A.P.D. and S.T.; Writing-review and editing, K.D.H., S.C.K., S.T. and S.L. All authors have read and agreed to the published version of the manuscript.

Funding: Saowaluck Tibpromma would like to thank the International Postdoctoral Exchange Fellowship Program (number Y9180822S1), CAS President's International Fellowship Initiative (PIFI) (number 2020PC0009), China Postdoctoral Science Foundation, and the Yunnan Human Resources, and Social Security Department Foundation for funding her postdoctoral research. Samantha C. Kaunarathna thanks CAS President's International Fellowship Initiative (PIFI) for funding his postdoctoral research (No. 2018PC0006) and the National Science Foundation of China (NSFC) for funding this work under the project code 31851110759. Kevin D. Hyde thanks the Thailand Research Funds for the grant "Impact of Climate Change on Fungal Diversity and Biogeography in the Greater Mekong Subregion (RDG6130001)". This work was partly supported by Chiang Mai University.

Institutional Review Board Statement: Not applicable.

Informed Consent Statement: Not applicable.

Data Availability Statement: Not applicable.

Acknowledgments: Sirinapa Konta is grateful to Paul Kirk, Shaun Pennycook, Saranyaphat Boonmee, and Sirilak Radbouchoom for their valuable suggestions and help.

Conflicts of Interest: The authors declare no conflict of interest.

\section{References}

1. Lewis, C.E.; Baker, W.J.; Asmussen, C.B. DNA and palm evolution. Palms 2000, 44, 19-24.

2. Hawksworth, D.L.; Lücking, R. Fungal diversity revisited: 2.2 to 3.8 million species. Fungal Kingd. 2017, 4, 79-95. [CrossRef]

3. Hyde, K.D.; Norphanphoun, C.; Maharachchikumbura, S.S.N.; Bhat, D.J.; Jones, E.B.G.; Bundhun, D.; Chen, Y.J.; Bao, D.F.; Boonmee, S.; Calabon, M.S.; et al. Refined families of Sordariomycetes. Mycosphere 2020, 11, 305-1059. [CrossRef]

4. Samarakoon, M.C.; Thongbai, B.; Hyde, K.D.; Brönstrup, M.; Beutling, U.; Lambert, C.; Miller, A.N.; Liu, J.K.J.; Promputtha, I.; Stadler, M. Elucidation of the life cycle of the endophytic genus Muscodor and its transfer into the genus Induratia in Induratiaceae fam. nov., based on a polyphasic taxonomic approach. Fungal Divers. 2020, 101, 177-210. [CrossRef] 
5. Senanayake, I.C.; Maharachchikumbura, S.S.N.; Hyde, K.D.; Bhat, J.D.; Jones, E.B.G.; McKenzie, E.H.C.; Dai, D.Q.; Daranagama, D.A.; Dayarathne, M.C.; Goonasekara, I.D.; et al. Towards unraveling relationships in Xylariomycetidae (Sordariomycetes). Fungal Divers. 2015, 73, 73-144. [CrossRef]

6. Maharachchikumbura, S.S.N.; Hyde, K.D.; Jones, E.G.; McKenzie, E.H.; Huang, S.K.; Abdel-Wahab, M.A.; Daranagama, D.A.; Dayarathne, M.; D'souza, M.J.; Goonasekara, I.D.; et al. Towards a natural classification and backbone tree for Sordariomycetes. Fungal Divers. 2015, 72, 199-301. [CrossRef]

7. Hongsanan, S.; Maharachchikumbura, S.S.N.; Hyde, K.D.; Samarakoon, M.C.; Jeewon, R.; Zhao, Q.; Al-Sadi, A.M.; Bahkali, A.H. An updated phylogeny of Sordariomycetes based on phylogenetic and molecular clock evidence. Fungal Divers. 2017, 84, 25-41. [CrossRef]

8. Wijayawardene, N.N.; Hyde, K.D.; Al-Ani, L.K.T.; Tedersoo, L.; Haelewaters, D.; Rajeshkumar, K.C.; Zhao, R.L.; Aptroot, A.; Leontyev, D.V.; Saxena, R.K.; et al. Outline of Fungi and fungus-like taxa. Mycosphere 2020, 11, 1060-1456. [CrossRef]

9. Krug, J.C. The genus Cainia and a new family, Cainiaceae. Sydowia 1978, 30, 122-133.

10. Maharachchikumbura, S.S.N.; Hyde, K.D.; Jones, E.G.; McKenzie, E.H.C.; Bhat, J.D.; Dayarathne, M.C.; Huang, S.K.; Norphanphoun, C.; Senanayake, I.C.; Perera, R.H.; et al. Families of Sordariomycetes. Fungal Divers. 2016, 79, 1-317. [CrossRef]

11. Mapook, A.; Hyde, K.D.; McKenzie, E.H.; Jones, E.G.; Bhat, D.J.; Jeewon, R.; Stadler, M.; Samarakoon, M.C.; Malaithong, M.; Tanunchai, B.; et al. Taxonomic and phylogenetic contributions to fungi associated with the invasive weed Chromolaena odorata (Siam weed). Fungal Divers. 2020, 101, 1-175. [CrossRef]

12. Hyde, K.D.; Norphanphoun, C.; Chen, J.; Dissanayake, A.J.; Doilom, M.; Hongsanan, S.; Jayawardena, R.S.; Jeewon, R.; Perera, R.H.; Thongbai, B.; et al. Thailand's amazing diversity-Up to $96 \%$ of fungi in northern Thailand are novel. Fungal Divers. 2018, 93, 215-239. [CrossRef]

13. Hyde, K.D.; Dong, Y.; Phookamsak, R.; Jeewon, R.; Bhat, D.J.; Jones, E.B.; Liu, N.G.; Abeywickrama, P.D.; Mapook, A.; Wei, D.P.; et al. Fungal diversity notes 1151-1276: Taxonomic and phylogenetic contributions on genera and species of fungal taxa. Fungal Divers. 2020, 100, 5-277. [CrossRef]

14. Hyde, K.D.; Jeewon, R.; Chen, Y.J.; Bhunjun, C.S.; Calabon, M.S.; Jiang, H.B.; Lin, C.G.; Norphanphoun, C.; Sysouphanthong, P.; Pem, D.; et al. The numbers of fungi: Is the descriptive curve flattening? Fungal Divers. 2020, 103, 219-271. [CrossRef]

15. Konta, S.; Hyde, K.D.; Eungwanichayapant, P.D.; Doilom, M.; Tennakoon, D.S.; Senwanna, C.; Boonmee, S. Fissuroma (Aigialaceae: Pleosporales) appears to be hyperdiverse on Arecaceae: Evidence from two new species from southern Thailand. Acta Bot. Bras. 2020, 34, 384-393. [CrossRef]

16. Senanayake, I.C.; Rathnayaka, A.R.; Marasinghe, D.S.; Calabon, M.S.; Gentekaki, E.; Lee, H.B.; Hurdeal, V.G.; Pem, D.; Dissanayake, L.S.; Wijesinghe, S.N.; et al. Morphological approaches in studying fungi: Collection, examination, isolation, sporulation and preservation. MYCOSP 2020, 11, 2678-2754. [CrossRef]

17. Jayasiri, S.C.; Hyde, K.D.; Ariyawansa, H.A.; Bhat, J.; Buyck, B.; Cai, L.; Dai, Y.C.; Abd-Elsalam, K.A.; Ertz, D.; Hidayat, I.; et al. The Faces of Fungi database: Fungal names linked with morphology, phylogeny and human impacts. Fungal Divers. 2015, 74, 3-18. [CrossRef]

18. Index Fungorum. 2021. Available online: http://www.indexfungorum.org/names/Names.asp (accessed on 30 April 2020).

19. Dissanayake, A.J.; Bhunjun, C.S.; Maharachchikumbura, S.S.N.; Liu, J.K. Applied aspects of methods to infer phylogenetic relationships amongst fungi. Mycosphere 2020, 11, 2652-2676. [CrossRef]

20. O'Donnell, K.; Cigelnik, E. Two divergent intragenomic rDNA ITS2 types within amonophyletic lineage of the fungus Fusarium are nonorthologous. Mol. Phylogenet. Evol. 1997, 7, 103-116. [CrossRef] [PubMed]

21. Pintos, Á.; Alvarado, P.; Planas, J.; Jarling, R. Six new species of Arthrinium from Europe and notes about A. caricicola and other species found in Carex spp. hosts. MycoKeys 2019, 49, 15-48. [CrossRef]

22. Crous, P.W.; Groenewald, J.Z. A phylogenetic re-evaluation of Arthrinium. IMA Fungus 2013, 4, 133-154. [CrossRef]

23. Wang, M.; Liu, F.; Crous, P.W.; Cai, L. Phylogenetic reassessment of Nigrospora: Ubiquitous endophytes, plant and human pathogens. Pers. Mol. Phylogeny Evol. Fungi 2017, 39, 118-142. [CrossRef] [PubMed]

24. Crous, P.W.; Schumacher, R.K.; Akulov, A.; Thangavel, R.; Hernández-Restrepo, M.; Carnegie, A.J.; Cheewangkoon, R.; Wingfield, M.J.; Summerell, B.A.; Quaedvlieg, W.; et al. New and interesting fungi. 2. Fungal Syst. Evol. 2019, 3, 57-134. [CrossRef] [PubMed]

25. Liu, F.; Bonthond, G.; Groenewald, J.Z.; Cai, L.; Crous, P.W. Sporocadaceae, a family of coelomycetous fungi with appendage-bearing conidia. Stud. Mycol. 2019, 92, 287-415. [CrossRef] [PubMed]

26. Crous, P.W.; Shivas, R.G.; Quaedvlieg, W.; van der Bank, M.; Zhang, Y.; Summerell, B.A.; Guarro, J.; Wingfield, M.J.; Wood, A.R.; Alfenas, A.C.; et al. Fungal Planet description sheets: 214-280. Pers. Mol. Phylogeny Evol. Fungi 2014, 32, 184-306. [CrossRef] [PubMed]

27. Jeewon, R.; Liew, E.C.; Hyde, K.D. Molecular systematics of the Amphisphaeriaceae based on cladistic analyses of partial LSU rDNA gene sequences. Mycol. Res. 2003, 107, 1392-1402. [CrossRef]

28. Liu, J.K.; Hyde, K.D.; Jones, E.G.; Ariyawansa, H.A.; Bhat, D.J.; Boonmee, S.; Maharachchikumbura, S.S.N.; McKenzie, E.H.C.; Phookamsak, R.; Phukhamsakda, C.; et al. Fungal diversity notes 1-110: Taxonomic and phylogenetic contributions to fungal species. Fungal Divers. 2015, 72, 1-197. [CrossRef]

29. Jaklitsch, W.M.; Gardiennet, A.; Voglmayr, H. Resolution of morphology-based taxonomic delusions: Acrocordiella, Basiseptospora, Blogiascospora, Clypeosphaeria, Hymenopleella, Lepteutypa, Pseudapiospora, Requienella, Seiridium and Strickeria. Pers. Mol. Phylogeny Evol. Fungi 2016, 37, 82-105. [CrossRef] 
30. Tibpromma, S.; Hyde, K.D.; Jeewon, R.; Maharachchikumbura, S.S.; Liu, J.K.; Bhat, D.J.; Jones, E.G.; McKenzie, E.H.; Camporesi, E.; Bulgakov, T.S.; et al. Fungal diversity notes 491-602: Taxonomic and phylogenetic contributions to fungal taxa. Fungal Divers. 2017, 83, 1-261. [CrossRef]

31. Lumbsch, H.T.; Schmitt, I.; Lindemuth, R.; Miller, A.; Mangold, A.; Fernandez, F.; Huhndorf, S. Performance of four ribosomal DNA regions to infer higher-level phylogenetic relationships of inoperculate euascomycetes (Leotiomyceta). Mol. Phylogenet. Evol. 2005, 34, 512-524. [CrossRef]

32. Okada, G.; Iida, T.; Ohkuma, M. The DNA Bank, RIKEN Bio Resource Research Center, Japan, 2017. Available online: https: //www.jcm.riken.jp/cgi-bin/jcm/jcm_number?JCM=7946 (accessed on 30 April 2020).

33. Bhattacharya, D.; Lutzoni, F.; Reeb, V.; Simon, D.; Nason, J.; Fernandez, F. Widespread occurrence of spliceosomal introns in the rDNA genes of ascomycetes. Mol. Biol. Evol. 2000, 17, 1971-1984. [CrossRef]

34. Voglmayr, H.; Friebes, G.; Gardiennet, A.; Jaklitsch, W.M. Barrmaelia and Entosordaria in Barrmaeliaceae (fam. nov., Xylariales) and critical notes on Anthostomella-like genera based on multigene phylogenies. Mycol. Prog. 2018, 17, 155-177. [CrossRef] [PubMed]

35. Asgari, B.; Zare, R. A contribution to the taxonomy of the genus Coniocessia (Xylariales). Mycol. Prog. 2011, 10, 189-206. [CrossRef]

36. García, D.; Stchigel, A.M.; Cano, J.; Calduch, M.; Hawksworth, D.L.; Guarro, J. Molecular phylogeny of Coniochaetales. Mycol. Res. 2006, 110, 1271-1289. [CrossRef] [PubMed]

37. Wanasinghe, D.N.; Phukhamsakda, C.; Hyde, K.D.; Jeewon, R.; Lee, H.B.; Jones, E.G.; Tibpromma, S.; Tennakoon, D.S.; Dissanayake, A.J.; Jayasiri, S.C.; et al. Fungal diversity notes 709-839: Taxonomic and phylogenetic contributions to fungal taxa with an emphasis on fungi on Rosaceae. Fungal Divers. 2018, 89, 1-236. [CrossRef]

38. Senwanna, C.; Phookamsak, R.; Doilom, M.; Hyde, K.D.; Cheewangkoon, R. Novel taxa of Diatrypaceae from Para rubber (Hevea brasiliensis) in northern Thailand; introducing a novel genus Allocryptovalsa. Mycosphere 2017, 8, 1835-1855. [CrossRef]

39. Konta, S.; Maharachchikumbura, S.S.N.; Senanayake, I.C.; McKenzie, E.H.C.; Stadler, M.; Boonmee, S.; Phookamsak, R.; Jayawardena, R.S.; Senwanna, C.; Hyde, K.D.; et al. A new genus Allodiatrype, five new species and a new host record of diatrypaceous fungi from palms (Arecaceae). Mycosphere 2020, 11, 239-268. [CrossRef]

40. Jaklitsch, W.M.; Fournier, J.; Rogers, J.D.; Voglmayr, H. Phylogenetic and taxonomic revision of Lopadostoma. Pers. Mol. Phylogeny Evol. Fungi 2014, 32, 52-82. [CrossRef]

41. Spatafora, J.W.; Sung, G.H.; Johnson, D.; Hesse, C.; O’Rourke, B.; Serdani, M.; Spotts, R.; Lutzoni, F.; Hofstetter, V.; Miadlikowska, J.; et al. A five-gene phylogeny of Pezizomycotina. Mycologia 2006, 98, 1018-1028. [CrossRef] [PubMed]

42. Lynch, S.C.; Eskalen, A.; Zambino, P.J.; Mayorquin, J.S.; Wang, D.H. Identification and pathogenicity of Botryosphaeriaceae species associated with coast live oak (Quercus agrifolia) decline in southern California. Mycologia 2013, 105, 125-140. [CrossRef]

43. Rolshausen, P.E.; Mahoney, N.E.; Molyneux, R.J.; Gubler, W.D. A reassessment of the species concept in Eutypa lata, the causal agent of Eutypa dieback of grapevine. Phytopathology 2006, 96, 369-377. [CrossRef] [PubMed]

44. Vu, D.; Groenewald, M.; De Vries, M.; Gehrmann, T.; Stielow, B.; Eberhardt, U.; Al-Hatmi, A.; Groenewald, J.Z.; Cardinali, G.; Houbraken, J.; et al. Large-scale generation and analysis of filamentous fungal DNA barcodes boosts coverage for kingdom fungi and reveals thresholds for fungal species and higher taxon delimitation. Stud. Mycol. 2019, 92, 135-154. [CrossRef] [PubMed]

45. Acero, F.J.; González, V.; Sánchez-Ballesteros, J.; Rubio, V.; Checa, J.; Bills, G.F.; Salazar, O.; Platas, G.; Peláez, F. Molecular phylogenetic studies on the Diatrypaceae based on rDNA-ITS sequences. Mycologia 2004, 96, 249-259. [CrossRef] [PubMed]

46. Dayarathne, M.C.; Phookamsak, R.; Hyde, K.D.; Manawasinghe, I.S.; To-Anun, C.; Jones, G.E. Halodiatrype, a novel diatrypaceous genus from mangroves with $H$. salinicola and H. avicenniae spp. nov. Mycosphere 2016, 7, 612-627. [CrossRef]

47. Klaysuban, A.; Sakayaroj, J.; Jones, E.G. An additional marine fungal lineage in the Diatrypaceae, Xylariales: Pedumispora rhizophorae. Bot. Mar. 2014, 57, 413-420. [CrossRef]

48. Hyde, K.D.; Tennakoon, D.S.; Jeewon, R.; Bhat, D.J.; Maharachchikumbura, S.S.N.; Rossi, W.; Leonardi, M.; Lee, H.B.; Mun, H.Y.; Houbraken, J.; et al. Fungal diversity notes 1036-1150: Taxonomic and phylogenetic contributions on genera and species of fungal taxa. Fungal Divers. 2019, 96, 1-242. [CrossRef]

49. Hyde, K.D.; Norphanphoun, C.; Abreu, V.P.; Bazzicalupo, A.; Chethana, K.T.; Clericuzio, M.; Dayarathne, M.C.; Dissanayake, A.J.; Ekanayaka, A.H.; He, M.Q.; et al. Fungal diversity notes 603-708: Taxonomic and phylogenetic notes on genera and species. Fungal Divers. 2017, 87, 1-235. [CrossRef]

50. Wendt, L.; Sir, E.B.; Kuhnert, E.; Heitkämper, S.; Lambert, C.; Hladki, A.I.; Romero, A.I.; Luangsa-Ard, J.J.; Srikitikulchai, P.; Peršoh, D.; et al. Resurrection and emendation of the Hypoxylaceae, recognised from a multigene phylogeny of the Xylariales. Mycol. Prog. 2018, 17, 115-154. [CrossRef]

51. Zhang, N.; Castlebury, L.A.; Miller, A.N.; Huhndorf, S.M.; Schoch, C.L.; Seifert, K.A.; Rossman, A.Y.; Rogers, J.D.; Kohlmeyer, J.; Volkmann-Kohlmeyer, B.; et al. An overview of the systematics of the Sordariomycetes based on a four-gene phylogeny. Mycologia 2006, 98, 1076-1087. [CrossRef]

52. Stadler, M.; Læssøe, T.; Fournier, J.; Decock, C.; Schmieschek, B.; Tichy, H.V.; Peršoh, D. A polyphasic taxonomy of Daldinia (Xylariaceae). Stud. Mycol. 2014, 77, 1-143. [CrossRef]

53. Koukol, O.; Kelnarová, I.; Černý, K. Recent observations of sooty bark disease of sycamore maple in Prague (Czech Republic) and the phylogenetic placement of Cryptostroma corticale. For. Pathol. 2015, 45, 21-27. [CrossRef]

54. Pažoutová, S.; Šrůtka, P.; Holuša, J.; Chudíčková, M.; Kolařík, M. The phylogenetic position of Obolarina dryophila (Xylariales). Mycol. Prog. 2010, 9, 501-507. [CrossRef] 
55. Kuhnert, E.; Sir, E.B.; Lambert, C.; Hyde, K.D.; Hladki, A.I.; Romero, A.I.; Rohde, M.; Stadler, M. Phylogenetic and chemotaxonomic resolution of the genus Annulohypoxylon (Xylariaceae) including four new species. Fungal Divers. 2017, 85, 1-43. [CrossRef]

56. Triebel, D.; Peršoh, D.; Wollweber, H.; Stadler, M. Phylogenetic relationships among Daldinia, Entonaema, and Hypoxylon as inferred from ITS nrDNA analyses of Xylariales. Nova Hedwig 2005, 80, 25-43. [CrossRef]

57. Kuhnert, E.; Fournier, J.; Peršoh, D.; Luangsa-Ard, J.J.D.; Stadler, M. New Hypoxylon species from Martinique and new evidence on the molecular phylogeny of Hypoxylon based on ITS rDNA and $\beta$-tubulin data. Fungal Divers. 2014, 64, 181-203. [CrossRef]

58. Læssøe, T.; Srikitikulchai, P.; Jennifer, J.; Luangsa-Ard, D.; Stadler, M. Theissenia reconsidered, including molecular phylogeny of the type species T. pyrenocrata and a new genus Durotheca (Xylariaceae, Ascomycota). IMA Fungus 2013, 4, 57-69. [CrossRef] [PubMed]

59. Daranagama, D.A.; Liu, X.; Chamyuang, S.; Stadler, M.; Bahkali, A.; Hyde, K.D. Rhopalostroma brevistipitatum sp. nov. from Thailand with an extended generic description for Rhopalostroma. Phytotaxa 2015, 227, 229-242. [CrossRef]

60. Stadler, M.; Kuhnert, E.; Peršoh, D.; Fournier, J. The Xylariaceae as model example for a unified nomenclature following the “One Fungus-One Name" (1F1N) concept. Mycology 2013, 4, 5-21. [CrossRef]

61. Bitzer, J.; Læssøe, T.; Fournier, J.; Kummer, V.; Decock, C.; Tichy, H.V.; Piepenbring, M.; Peršoh, D.; Stadler, M. Affinities of Phylacia and the daldinoid Xylariaceae, inferred from chemotypes of cultures and ribosomal DNA sequences. Mycol. Res. 2008, 112, 251-270. [CrossRef]

62. Miller, A.N.; Vasilyeva, L.N.; Rogers, J.D. Chlorostroma subcubisporum gen. et sp. nov. and notes on the systematic position of Thuemenella cubispora. Sydowia 2007, 59, 138-147.

63. Stadler, M.; Flessa, F.; Rambold, G.; Peršoh, D.; Fournier, J.; Læssøe, T.; Chlebicki, A.; Lechat, C. Chemotaxonomic and phylogenetic studies of Thamnomyces (Xylariaceae). Mycoscience 2010, 51, 189-207. [CrossRef]

64. Voglmayr, H.; Aguirre-Hudson, M.B.; Wagner, H.G.; Tello, S.; Jaklitsch, W.M. Lichens or endophytes? The enigmatic genus Leptosillia in the Leptosilliaceae fam. nov. (Xylariales), and Furfurella gen. nov. (Delonicicolaceae). Pers. Mol. Phylogeny Evol. Fungi 2019, 42, 228-260. [CrossRef] [PubMed]

65. Zhang, C.L.; Wang, G.P.; Mao, L.J.; Komon-Zelazowska, M.; Yuan, Z.L.; Lin, F.C.; Druzhinina, I.S.; Kubicek, C.P. Muscodor fengyangensis sp. nov. from southeast China: Morphology, physiology and production of volatile compounds. Fungal Biol. Rev. 2010, 114, 797-808. [CrossRef]

66. Becerra-Hernández, C.I.; González, D.; De Luna, E.; Mena-Portales, J. First report of pleoanamorphy in Gyrothrix verticiclada with an Idriella-like synanamorph. Cryptogam. Mycol. 2016, 37, 241-252. [CrossRef]

67. Hernández-Restrepo, M.; Groenewald, J.Z.; Crous, P.W. Taxonomic and phylogenetic re-evaluation of Microdochium, Monographella and Idriella. Pers. Mol. Phylogeny Evol. Fungi 2016, 36, 57-82. [CrossRef]

68. Bundhun, D.; Jeewon, R.; Dayarathne, M.C.; Bulgakov, T.S.; Khramtsov, A.K.; Aluthmuhandiram, J.V.; Pem, D.; To-Anun, C.; Hyde, K.D. A morpho-molecular re-appraisal of Polystigma fulvum and P. rubrum (Polystigma, Polystigmataceae). Phytotaxa 2019, 422, 209-224. [CrossRef]

69. Maharachchikumbura, S.S.N.; Hyde, K.D.; Perera, R.H.; Al-Sadi, A.M. Acrocordiella omanensis sp. nov. (Requienellaceae, Xylariales) from the Sultanate of Oman. Phytotaxa 2018, 338, 294-300. [CrossRef]

70. Park, J.H.; Choi, G.J.; Lee, H.B.; Kim, K.M.; Jung, H.S.; Lee, S.W.; Jang, K.S.; Cho, K.Y.; Kim, J.C. Griseofulvin from Xylaria sp. strain F0010, an endophytic fungus of Abies holophylla and its antifungal activity against plant pathogenic fungi. J. Microbiol. Biotechnol. 2005, 15, 112-117.

71. Hsieh, H.M.; Lin, C.R.; Fang, M.J.; Rogers, J.D.; Fournier, J.; Lechat, C.; Ju, Y.M. Phylogenetic status of Xylaria subgenus Pseudoxylaria among taxa of the subfamily Xylarioideae (Xylariaceae) and phylogeny of the taxa involved in the subfamily. Mol. Phylogenet. Evol. 2010, 54, 957-969. [CrossRef]

72. Dai, D.Q.; Phookamsak, R.; Wijayawardene, N.N.; Li, W.J.; Bhat, D.J.; Xu, J.C.; Taylor, J.E.; Hyde, K.D.; Chukeatirote, E. Bambusicolous fungi. Fungal Divers. 2017, 82, 1-105. [CrossRef]

73. Marincowitz, S.; Crous, P.W.; Groenewald, J.Z.; Wingfield, M.J. Microfungi Occurring on Proteaceae in the Fynbos; CBS Biodiversity Series; CBS-KNAW Fungal Biodiversity Centre: Utrecht, The Netherlands, 2008.

74. Jaklitsch, W.M.; Voglmayr, H. Phylogenetic relationships of five genera of Xylariales and Rosasphaeria gen. nov. (Hypocreales). Fungal Divers. 2012, 52, 75-98. [CrossRef]

75. Johnston, P.R.; Rogers, J.D.; Park, D.; Martin, N.A. Entalbostroma erumpens gen. et sp. nov. (Xylariaceae) from Phormium in New Zealand. Mycotaxon 2016, 131, 765-771. [CrossRef]

76. Soca-Chafre, G.; Rivera-Orduña, F.N.; Hidalgo-Lara, M.E.; Hernandez-Rodriguez, C.; Marsch, R.; Flores-Cotera, L.B. Molecular phylogeny and paclitaxel screening of fungal endophytes from Taxus globosa. Fungal Biol. Rev. 2011, 115, 143-156. [CrossRef]

77. Jayanetti, D.R.; Yue, Q.; Bills, G.F.; Gloer, J.B. Hypocoprins A-C: New sesquiterpenoids from the coprophilous fungus Hypocopra rostrata. J. Nat. Prod. 2015, 78, 396-401. [CrossRef]

78. Ju, Y.M.; Hsieh, H.M.; Ho, M.C.; Szu, D.H.; Fang, M.J. Theissenia rogersii sp. nov. and phylogenetic position of Theissenia. Mycologia 2007, 99, 612-621. [CrossRef] [PubMed]

79. Voglmayr, H.; Beenken, L. Linosporopsis, a new leaf-inhabiting scolecosporous genus in Xylariaceae. Mycol. Prog. 2020, 19, 205-222. [CrossRef] 
80. U’Ren, J.M.; Miadlikowska, J.; Zimmerman, N.B.; Lutzoni, F.; Stajich, J.E.; Arnold, A.E. Contributions of North American endophytes to the phylogeny, ecology, and taxonomy of Xylariaceae (Sordariomycetes, Ascomycota). Mol. Phylogenet. Evol. 2016, 98, 210-232. [CrossRef]

81. Konta, S.; Hyde, K.D.; Phookamsak, R.; Xu, J.C.; Maharachchikumbura, S.S.N.; Daranagama, D.A.; McKenzie, E.H.C.; Boonmee, S.; Tibpromma, S.; Eungwanichayapant, P.D.; et al. Polyphyletic genera in Xylariaceae (Xylariales): Neoxylaria gen. nov. and Stilbohypoxylon. Mycosphere 2020, 11, 2629-2651. [CrossRef]

82. Tang, A.M.C.; Jeewon, R.; Hyde, K.D. A re-evaluation of the evolutionary relationships within the Xylariaceae based on ribosomal and protein-coding gene sequences. Fungal Divers. 2009, 34, 127-155.

83. Fournier, J.; Stadler, M.; Hyde, K.D.; Duong, M.L. The new genus Rostrohypoxylon and two new Annulohypoxylon species from Northern Thailand. Fungal Divers. 2010, 40, 23-36. [CrossRef]

84. Dai, D.Q.; Bahkali, A.H.; Li, Q.R.; Bhat, D.J.; Wijayawardene, N.N.; Li, W.J.; Chukeatirote, E.; Zhao, R.L.; Xu, J.C.; Hyde, K.D. Vamsapriya (Xylariaceae) re-described, with two new species and molecular sequence data. Cryptogam. Mycol. 2014, 35, 339-357. [CrossRef]

85. Jiang, H.B.; Phookamsak, R.; Bhat, D.J.; Khan, S.; Bahkali, A.; Elgorban, A.; Hyde, K.D. Vamsapriya yunnana, a new species of Vamsapriya (Xylariaceae, Xylariales) associated with bamboo from Yunnan, China. Phytotaxa 2018, 356, 61-70. [CrossRef]

86. Nonaka, K.; Ishii, T.; Shiomi, K.; Ōmura, S.; Masuma, R. Virgaria boninensis, a new hyphomycete (Xylariaceae) from soils in the Bonin Islands, Japan. Mycoscience 2013, 54, 394-399. [CrossRef]

87. Sir, E.B.; Kuhnert, E.; Surup, F.; Hyde, K.D.; Stadler, M. Discovery of new mitorubrin derivatives from Hypoxylon fulvo-sulphureum sp. nov. (Ascomycota, Xylariales). Mycol. Prog. 2015, 14, 28. [CrossRef]

88. Cheng, X.; Li, W.; Cai, L. Molecular phylogeny of Ascotricha, including two new marine algae-associated species. Mycologia 2015, 107, 490-504. [CrossRef]

89. Daranagama, D.A.; Hyde, K.D.; Sir, E.B.; Thambugala, K.M.; Tian, Q.; Samarakoon, M.C.; McKenzie, E.H.; Jayasiri, S.C.; Tibpromma, S.; Bhat, J.D. Towards a natural classification and backbone tree for Graphostromataceae, Hypoxylaceae, Lopadostomataceae and Xylariaceae. Fungal Divers. 2018, 88, 1-165. [CrossRef]

90. Crous, P.W.; Schumacher, R.K.; Wingfield, M.J.; Lombard, L.; Giraldo, A.; Christensen, M.; Gardiennet, A.; Nakashima, C.; Pereira, O.L.; Smith, A.J. Fungal systematics and evolution: FUSE 1. Sydowia 2015, 67, 81-118. [CrossRef]

91. Li, D.W.; Schultes, N.P.; Chen, J.Y.; Wang, Y.X.; Castañeda-Ruiz, R.F. Circinotrichum sinense, a new asexual fungus from Hubei, China. Botany 2017, 95, 1099-1108. [CrossRef]

92. Crous, P.W.; Wingfield, M.J.; Lombard, L.; Roets, F.; Swart, W.J.; Alvarado, P.; Carnegie, A.J.; Moreno, G.; Luangsaard, J.; Thangavel, R.; et al. Fungal Planet description sheets: 951-1041. Pers. Mol. Phylogeny Evol. Fungi 2019, 43, 223-425. [CrossRef]

93. Li, J.F.; Phookamsak, R.; Jeewon, R.; Tibpromma, S.; Maharachchikumbura, S.S.N.; Bhat, D.J.; Chukeatirote, E.; Lumyong, S.; Hyde, K.D.; McKenzie, E.H.C. Establishment of Zygosporiaceae fam. nov. (Xylariales, Sordariomycetes) based on rDNA sequence data to accommodate Zygosporium. MYCOAP 2017, 8, 1855-1868. [CrossRef]

94. Katoh, K.; Standley, K. MAFFT Multiple Sequence Alignment Software Version 7: Improvements in performance and usability. Mol. Biol. Evol. 2013, 30, 772-780. [CrossRef] [PubMed]

95. Kumar, S.; Stecher, G.; Tamura, K. MEGA7: Molecular evolutionary genetics analysis version 7.0 for bigger datasets. Mol. Biol. Evol. 2016, 33, 1870-1874. [CrossRef] [PubMed]

96. Glez-Peña, D.; Gómez-Blanco, D.; Reboiro-Jato, M.; Fdez-Riverola, F.; Posada, D. ALTER: Program-oriented conversion of DNA and protein alignments. Nucleic Acid. Res. 2010, 38, 14-18. [CrossRef] [PubMed]

97. Nylander, J.A.A. MrModeltest, Version 2; Program Distributed by the Author; Evolutionary Biology Centre, Uppsala University: Uppsala, Sweden, 2004; Available online: https:/ / github.com/Nylander (accessed on 30 April 2020).

98. Rambaut, A. FigTree Version 1.4.0. 2012. Available online: http://tree.bio.ed.ac.uk/software/figtree/ (accessed on 30 April 2020).

99. Daranagama, D.A.; Camporesi, E.; Tian, Q.; Liu, X.; Chamyuang, S.; Stadler, M.; Hyde, K.D. Anthostomella is polyphyletic comprising several genera in Xylariaceae. Fungal Divers. 2015, 73, 203-238. [CrossRef]

100. Petch, T. The genus Endocalyx, Berkeley and Broome. Ann. Bot. 1908, 22, 389-400. [CrossRef]

101. Okada, G.; Tubaki, K. A new species and a new variety of Endocalyx (Deuteromycotina) from Japan. Mycologia 1984, 76, 300-313. [CrossRef]

102. Vitoria, N.S.; Cavalcanti, M.A.Q.; Luz, E.D.M.N.; Bezerra, J.L. Endocalyx melanoxanthus var. melanoxanthus (Ascomycota): New to Brazil and three new hosts. Mycotaxon 2011, 117, 109-113. [CrossRef]

103. Patel, U.S.; Pandey, A.K.; Rajak, R.C. Two new hyphomycetes. J. Mycol. Plant Pathol. 2002, 32, 70-71.

104. Kobayashi, T. Index of Fungi Inhabiting Woody Plants in Japan: Host, Distribution and Literature; Zenkoku-Noson-Kyoiku Kyokai Publishing Co., Ltd.: Tokyo, Japan, 2007; pp. 1-1227.

105. Lu, B.; Hyde, K.D.; Ho, W.H.; Tsui, K.M.; Taylor, J.E.; Wong, K.M.; Yanna; Zhou, D. Checklist of Hong Kong Fungi; Fungal Diversity Press: Hong Kong, China, 2000; pp. 1-207.

106. Portales, J.M.; Sierra, A.M. Nuevas especies de Endocalyx y Stachylidium (Hyphomycetes, Deuteromycotina) de Cuba. Rev. Jard. Bot. Nac. 1984, 5, 53-60.

107. Kapoor, J.N.; Munjal, R.L. Indian species of Stilbaceae. Indian Phytopathol. 1966, 19, 348-350.

108. Heredia, G.; Arias, R.M.; Reyes, M. Contribucion al conocimiento de los hongos Hyphomycetes de Mexico. Acta Bot. Mex. 2000, 51, 39-51. [CrossRef] 
109. Taylor, J.E.; Hyde, K.D. Microfungi of Tropical and Temperate Palms; Fungal Diversity Press: Hong Kong, China, 2003 ; pp. 1-459.

110. Hughes, S.J. Fungi from the gold coast. I. Mycol. Pap. 1952, 48, 1-91.

111. Anonymous. Index of Plant Diseases in the United States. USDA Agric. Handb. 1960, 165, 1-531.

112. Raabe, R.D.; Conners, I.L.; Martinez, A.P. Checklist of Plant Diseases in Hawaii; Information Text Series No. 22; College of Tropical Agriculture and Human Resources, University of Hawaii: Honolulu, HI, USA, 1981; pp. 1-313.

113. Turner, P.D. Microorganisms associated with oil palm (Elaeis guineensis Jacq.). Phytopathol. Pap. 1971, 14, 1-58.

114. Shaw, D.E. Microorganisms in Papua New Guinea. Dept. Prim. Ind. Res. Bull. 1984, 33, 1-344.

115. Urtiaga, R. [Host index of plant diseases and disorders from Venezuela-Addendum]. 2004; pp. 1-268. Available online: https: //nt.ars-grin.gov/fungaldatabases/fungushost/new_rptOneLit.cfm?fungRec=39196\&thisError= (accessed on 30 April 2020).

116. Urtiaga, R. Indice de enfermedades en plantas de Venezuela y Cuba, Second Edition. 2004; pp. 1-301. Available online: https: //nt.ars-grin.gov/fungaldatabases / fungushost/new_rptOneLit.cfm?fungRec=39195\&thisError= (accessed on 30 April 2020).

117. Thaung, M.M. A list of hypomycetes (and agonomycetes) in Burma. Australas. Mycol. 2008, 27, 149-172.

118. Pinruan, U.; Hyde, K.D.; Lumyong, S.; McKenzie, E.H.C.; Jones, E.B.G. Occurrence of fungi on tissues of the peat swamp palm Licuala longicalycata. Fungal Divers. 2007, 25, 157-173.

119. Matsushima, T. Matsushima Mycological Memoirs No. 1. Saprophytic Microfungi from Taiwán, Part1; Matsushima Fungus collection: Kobe, Japan, 1980; pp. 1-82.

120. Ahmad, S. Fungi of West Pakistan. Biol. Soc. Pak. Monogr. 1969, 5, 1-110.

121. Zhuang, W.Y. Higher Fungi of Tropical China; Mycotaxon Limited: Ithaca, NY, USA, 2001; pp. 1-485.

122. Hughes, S.J. New Zealand Fungi. 25. Miscellaneous species. N. Z. J. Bot. 1978, 16, 311-370. [CrossRef]

123. Delgado, G. South Florida microfungi: New records of saprophytic hyphomycetes on plant debris. FLA Sci. 2008, 71, 76-89.

124. Arnold, G.R.W. Lista de Hongos Fitopatogenos de Cuba; Ministerio de Cultura Editorial Cientifico-Tecnica: Havana, Cuba, 1986; pp. 1-207.

125. Sierra, A.M. Hifomicetes Demaciaceos de Sierra del Rosario, Cuba; Editorial Academica: Havana, Cuba, 1984; pp. 1-81.

126. Urtiaga, R. Indice de Enfermedades en Plantas de Venezuela y Cuba; Impresos en Impresos Nuevo Siglo S.R.L.: Barquisimeto, Venezuela, 1986; pp. 1-202.

127. Delgado-Rodriguez, G.; Mena-Portales, J.; Calduch, M.; Decock, C. Hyphomycetes (hongos mitosporicos) del area protegida mil cumbres, Cuba Occidental. Cryptog. Mycol. 2002, 23, 277-293.

128. Petrak, F. Ein Beitrag zur Pilzflora Floridas. Sydowia 1953, 7, 103-116.

129. Sutton, B.C. New and interesting Hyphomycetes from Tampa, Florida. Mycologia 1978, 70, 784-801. [CrossRef]

130. Capdeet, M.; Romero, A.I. Fungi from palms in Argentina. 1. Mycotaxon 2010, 112, 339-355. [CrossRef]

131. Vitoria, N.S.; Fortes, N.G.S.; dos Santos, M.A.L.; Barbosa, R.L. Mycota (Ascomycota) of Syagrus coronata (Mart.) Becc., Raso da Catarina Ecological Station, Brazil: New records. Acta Bras. 2020, 4, 110-120. [CrossRef]

132. Hughes, S.J. Fungi from the gold coast. II. Mycol. Pap. 1953, 50, 1-104.

133. Ellis, M.B. Dematiaceous Hyphomycetes; Commonwealth Mycological Institute: London, UK, 1971; pp. 1-608.

134. Species Fungorum. 2021. Available online: http:/ / www.speciesfungorum.org/Names/Names.asp (accessed on 30 April 2020).

135. Samarakoon, B.C.; Wanasinghe, D.N.; Samarakoon, M.C.; Phookamsak, R.; McKenzie, E.H.; Chomnunti, P.; Hyde, K.D.; Lumyong, S.; Karunarathna, S.C. Multi-gene phylogenetic evidence suggests Dictyoarthrinium belongs in Didymosphaeriaceae (Pleosporales, Dothideomycetes) and Dictyoarthrinium musae sp. nov. on Musa from Thailand. MycoKeys 2020, 71, 101-118. [CrossRef] 